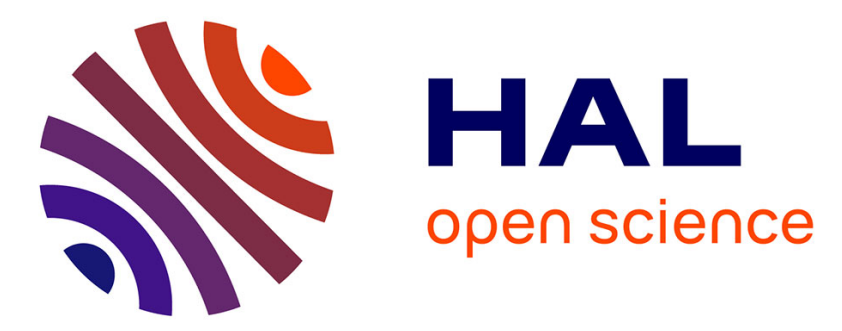

\title{
Homogenization of Solute Transport in Unsaturated Double-Porosity Media: Model and Numerical Validation
}

T.D. Tran Ngoc, N. H. N. Le, T. V. Tran, Azita Ahmadi-Senichault, Henri Bertin

\section{To cite this version:}

T.D. Tran Ngoc, N. H. N. Le, T. V. Tran, Azita Ahmadi-Senichault, Henri Bertin. Homogenization of Solute Transport in Unsaturated Double-Porosity Media: Model and Numerical Validation. Transport in Porous Media, 2020, 132 (1), pp.53-81. 10.1007/s11242-020-01380-6 . hal-03000736

\section{HAL Id: hal-03000736 https://hal.science/hal-03000736}

Submitted on 23 Jul 2021

HAL is a multi-disciplinary open access archive for the deposit and dissemination of scientific research documents, whether they are published or not. The documents may come from teaching and research institutions in France or abroad, or from public or private research centers.
L'archive ouverte pluridisciplinaire HAL, est destinée au dépôt et à la diffusion de documents scientifiques de niveau recherche, publiés ou non, émanant des établissements d'enseignement et de recherche français ou étrangers, des laboratoires publics ou privés. 


\title{
Homogenization of Solute Transport in Unsaturated Double-Porosity Media: Model and Numerical Validation
}

\author{
T. D. Tran Ngoc ${ }^{1} \cdot$ Nam H. N. Le ${ }^{2}$ Tieng V. Tran ${ }^{3} \cdot$ Azita Ahmadi $^{4} \cdot$ Henri Bertin $^{5}$
}

\begin{abstract}
The development of a macroscopic model for solute transport coupled with unsaturated water flow in double-porosity media is presented in this work, by using the asymptotic homogenization method. The model was derived for the case in which the medium exhibits a strong contrast of transport properties by upscaling rigorously the transport mechanisms from micro-scale to macro-scale. It consists of two coupled equations for dispersion-convection processes at macroscopic level and diffusion intervention from local scale that can be described by a non-Fickian behaviour of solute concentration breakthrough. The proposed model was numerically implemented in the environment of a finite element code (commercial software) and applied to $2 \mathrm{D}$ examples with different boundary conditions. To validate, a comparative analysis between the results obtained from the homogenized model and the fine scale model (reference solution obtained from explicit heterogeneous representation of the medium structure) was carried out. The obtained numerical tool for the two-scale implementation enables treating various types of two-equation models to study the macroscopic non-Fickian transport and also non-equilibrium evolution of concentration fields inside the micro-porous medium.
\end{abstract}

Keywords Non-Fickian transport · Double-porosity medium · Asymptotic homogenization $\cdot$ Modelling $\cdot$ Two-scale numerical simulation

T. D. Tran Ngoc

tntdung@hcmut.edu.vn

1 Centre Asiatique de Recherche sur l'Eau (CARE)/Faculty of Environment and Natural Resources, Ho Chi Minh City University of Technology, VNU-HCM, 268 Ly Thuong Kiet St., Dist. 10, Ho Chi Minh City, Viet Nam

2 CARE/Faculty of Geology and Petroleum Engineering, Ho Chi Minh City University of Technology, VNU-HCM, 268 Ly Thuong Kiet St., Dist. 10, Ho Chi Minh City, Viet Nam

3 Faculty of Civil Engineering, Ho Chi Minh City University of Technology and Education, 1 Vo Van Ngan St., Thu Duc Dist., Ho Chi Minh City, Viet Nam

4 Arts et Métiers ParisTech, CNRS, I2M Bordeaux, Esplanade des Arts et Métiers, 33405 Talence, France

5 I2M, UMR CNRS 5295, Université de Bordeaux, Esplanade des Arts et Métiers, 33405 Talence, France 


\section{Introduction}

The study of solute transport in porous media has not been declining, particularly in the actual context of exploitation and conservation of terrestrial resources. The motivation is stimulated by the fact that complex flow and transport processes are observed in different civil/industrial activities such as groundwater use, soil decontamination, oil/gas reservoirs or carbon dioxide geological sequestration (Doughty and Moridis 2018). Heterogeneity of the geological environment at various scales within the context of multiphase-multicomponent transport plays a key role on this complexity of the processes (e.g. Dagan 1984; Sudicky 1986; Dagan et al. 1990; Berkowitz et al. 2000; de Marsily et al. 2005; Niessner and Helmig 2007; Tran Ngoc et al. 2011; Gensterblum et al. 2015; Cremer et al. 2016; Wu et al. 2017; Portois et al. 2018). For such media, conventional models are not always appropriate to describe their non-standard behaviour. Non-Fickian transport assigned by an early breakthrough and tailing of solute concentration evolution in time and space throughout heterogeneous media has been much investigated in the literature (Berkowitz et al. 2008; Berkowitz and Scher 2009; Tran Ngoc et al. 2014). Nevertheless, the macroscopic non-Fickian transport taking into account the diffusion effect at the local scale needs further investigation (Dartois et al. 2018). These non-Fickian features are attributed to preferential flow transport implying local nonequilibrium concentrations inside the media (Sternberg et al. 1996; Lewandowska et al. 2004; Jarvis et al. 2016). The molecular diffusion ceases apparently to play a role with regard to the dispersion when the Péclet number $(\mathrm{Pe})$ is high enough (Fried and Combarnous 1971). In fact, Jankovic et al. (2009) proposed to neglect diffusion for weak to moderately heterogeneous porous media, but found that diffusion impacts considerably the transport for highly heterogeneous ones, through numerical simulations with $P e=O\left(10^{2}-10^{4}\right)$. The particular case of $P e=O(1)$ has to be more investigated for the question of the impact of local diffusion on the transport in unsaturated porous media.

The Barenblatt concept of the "double-porosity" medium (Barenblatt et al. 1960) can be used to replace a class of heterogeneous porous media (aggregated soils, fractured porous rocks, for example) in which a strong contrast in the local pore size characteristics is manifested. This concept of two overlapping sub-domains has been applied to phenomenological approach allowing to explain the non-conventional transport behaviour and to resolve practice problems. Using a double-porosity model revealed very appropriate for the granite basement reservoir of the White Tiger oilfield in Vietnam offshore (Ngo 2002; Ha et al. 2015), for example. Since the first models (Barenblatt et al. 1960; Warren and Root 1963), many continuum two-domain models (macroporosity and micro-porosity) were proposed with their mathematical form of two equations representing the transport mechanism in each domain and linked by an exchange term. Different models describing the unsaturated preferential flow and transport in double-porosity soils were reviewed by Šimůnek et al. (2003), Gerke (2006) and Köhne et al. (2009) in a comparative manner between them. The macroscopic equations proposed in these works were different one from another according to the imposed assumptions. It can be noticed that the complexity of the models, i.e. number of model parameters, increases in the respective order of the mobile-immobile model (van Genuchten and Wierenga 1976; Gaudet et al. 1977), the mobile-mobile model (Gerke and van Genuchten 1993) and the dual-permeability mobile-immobile model (Šimůnek and van Genuchten 2008). Moreover, the macroscopic form of all these models displays a coexistence of two concentration fields linked by a first-order mass transfer between the two 
porosity domains. It must be noted that this coefficient is only determined by the fitting of solute evolution curves (de Vries et al. 2017).

Upscaling approaches have contributed to a great advance in modelling several physical mechanisms including solute transport in double-porosity media. Among others, the asymptotic homogenization approach (Sanchez-Palencia 1980; Auriault 1983; Auriault et al. 2009) and the volume averaging method (Whitaker 1983; Quintard and Whitaker 1988; Whitaker 1999) have been applied to double-porosity transport issues thus improving profoundly the understanding of these phenomena thanks to the rigorous processes of upscaling from a small scale to a larger scale (Royer and Boutin 2012; Davit and Quintard 2017). One of the advantages of the upscaling method is to save resources when resolving macroscopic problems taking into account the microstructures of the medium through effective properties, instead of directly computing at a finer scale using a large number of mesh elements (Auriault 1991). This can be carried out for the case in which a representative elementary volume (REV) of the microstructure exists in the heterogeneous medium. It is not simple to obtain a REV, but using advanced geophysics and non-invasive imaging techniques to detect heterogeneities allows to establish the possible REV (Bear 1972; Jarvis et al. 2016).

Using the method of volume averaging Ahmadi et al. (1998) described the solute transport in saturated two-domain media by the generalized two-equation model. Estimating the first-order mass transfer term of this model is comparable to one of the aforementioned phenomenological models (Cherblanc et al. 2003). Cherblanc et al. (2007) compared the results of the two-equation model with those of fine scale simulations (porous or Darcy scale) for the cases of local-scale dispersion in which different permeability contrasts of the periodic two-domain medium (nodular system) in a numerical transport experiment were considered, but no diffusivity contrast was considered in their work. While providing a good prediction for the "mobile-mobile" system medium, a discrepancy with the "mobile-immobile" model was observed due to the estimation of mass transfer coefficient. Golfier et al. (2007) attempted to reproduce numerically the transport experiments in highly heterogeneous porous medium of Zinn et al. (2004). They proposed a mixed model derived from the two-equation model to confront with the experiments in considering a "mobile-immobile" system where only diffusion process occurs in the immobile domain and the full mass transport is maintained in the mobile domain. A reasonably good agreement between the experimental data and theory could only be obtained when accepting that the mass transfer coefficient had to be computed from direct solution at the local scale.

Various models have been developed for water flow and solute transport in doubleporosity media by using the asymptotic homogenization method of multiple-scale asymptotic expansions (Royer et al. 1996, 2002; Peszynska and Showalter 2007). A macroscopic model of diffusion-convection containing a diffusion equation in the micro-porosity domain was developed by Hornung (1991) under unsaturated condition. Auriault and Lewandowska (1995) developed the double-diffusivity model allowing to capture the tailing effect. Mikelic and Rosier (2004) derived a transport model by convection and dispersion in the macroporosity domain, coupled with adsorption at the interface of micro- and macro-pore regions, but without solute transport in the micro-pore region. In these models, Richard's equation and convection-diffusion/dispersion equation were employed to describe the transient flow and transport in the microstructure. Considering different characteristic times, different models described the steady-state double-porosity transport in saturated porous media (Tejchman 2004). Lewandowska et al. (2004) derived a model of the transient flow in a double-porosity medium of which the two porous materials, at the local scale, are saturated by water and air composing a macro-pore network surrounded by a micro-porous matrix. This 
highly hydraulic contrasted double-porosity medium with predefined dimensionless numbers characterizing the physical processes leads to conclude on the existence of flow only in the macro-porosity domain. This was successfully validated by experiments of different hydrologic situations in a physical double-porosity column (Lewandowska et al. 2005, 2008; Szymkiewicz et al. 2008). Associated with this model, a double-porosity dispersion-convection model has been developed in Tran Ngoc et al. (2011), taking into account the dispersion regime at the local scale (Andricevic 1998; Fiori 2001). It was validated by the unsaturated double-porosity experiments in which the salt diffusion coefficients in sand and clay (two material constituents of double-porosity medium) are different by only one order of magnitude (Tran Ngoc 2008). Based on the characteristic time analysis at three consecutive scales: from micro-pore scale to micro-porous scale and to macroscopic one, Royer and Boutin (2012) revised the theory for different flow and transport macroscopic behaviours in saturated double-porosity media. They found that the non-Fickian transport may occur for the cases of low diffusivity contrast. From a semi-analytical mean-field approximation, Brassart and Stainier (2019) obtained a homogenization-based diffusion model enabling to describe the long-tail effect for the case of large contrast of diffusivities in a two-phase composite. To go further, it is also interesting to develop a dispersive model for the case of a strong diffusivity contrast between two porous domains inside the unsaturated double-porosity medium, in order to pursue the study of the non-Fickian transport with memory effect for this case. Thus, this will allow us to fill in the double-porosity model catalogue obtained by the homogenization methods and to distinguish various models and to help the selection of a model appropriate for each particular application.

Numerical solutions for the two-equation model type have been developed (Pruess and Narasimhan 1982; Huyakorn et al. 1983; Gaudet et al. 1976; Ahmadi et al. 1998; Lewandowska et al. 2004; Cherblanc et al. 2007; Szymkiewicz 2013), since the first analytical solutions of Barenblatt et al. (1960), Barenblatt (1963), Warren and Root (1963) and Coats and Smith (1964). The complexity of resolving the two-domain transport resides in the fully transient problem of the mass transfer coupling between two concentrations at the macroscopic and microscopic scales. Thus, Golfier et al. (2007) stated "that begins to be nearly as difficult (if not more so) than the direct solution to the problem at the microscale". It is interesting to reproduce numerically many transport experiments performed in double-porosity media presented in the literature, not only for investigating the macroscopic behaviour but also for the study of transport processes at the microscopic scale (Zinn et al. 2004; Dalla Costa 2007; Ngien et al. 2012; Majdalani et al. 2015; Peng et al. 2015, for example). A powerful numerical tool is needed to simulate these experimental results.

The aim of this paper is to present (1) a development of a macroscopic transport model by dispersion-convection with local diffusion in a double-porosity medium presenting strong contrast of both flow and transport properties under unsaturated steadystate conditions, by using the asymptotic homogenization and (2) a numerical tool to implement the obtained mathematical model for macroscopic boundary value problems. The development of the model will start from the description of physical processes at the microscopic scale (local scale) and will be accompanied with the definition of the effective properties. The macroscopic model is solved in 2D numerical experiments performed by using a commercial FEM code. To validate the obtained model, comparison between theoretical predictions of the macroscopic model and reference solutions of the transport in heterogeneous finely discretized model of the experiments at the microscopic level is conducted. A discussion of the developed model and the compared results will be addressed. 


\section{Mathematical Formulation of the Problem}

In this section, we present the general conditions and physical situations in which the double-porosity dispersion-convection model will be derived in the framework of the asymptotic homogenization method.

\subsection{Double-Porosity Medium and Its Physical Characteristics}

The macroscopic double-porosity medium with characteristic length $L[\mathrm{~L}]$ considered as the same in Lewandowska et al. (2004) is a periodic porous medium $(\Omega)$ whose microstructure, i.e. a period with characteristic length $\ell[\mathrm{L}]$, contains two porous domains $\left(\Omega_{1}\right.$ and $\Omega_{2}$ ) of strongly contrasted physical properties (pore size, hydraulic permeability and transport diffusivity), distinguished by the common interface $\Gamma$ (Fig. 1). The ratio between these two characteristic lengths

$$
\varepsilon=\frac{\ell}{L}
$$

is an important small parameter for the upscaling technique. The condition $\varepsilon<<1$ is equivalent to the existence of a REV (Auriault 1991). The basic problem is to study the asymptotics of the solution as $\varepsilon \rightarrow 0$. We assume that the double-porosity medium is unsaturated with given constant water contents in the porous domain 1 (macro-porous ingredient $\left.\Omega_{1}\right), \theta_{1}\left[\mathrm{~L}^{3} / \mathrm{L}^{3}\right]$ and in the porous domain 2 (micro-porous ingredient $\Omega_{2}$ ), $\theta_{2}\left[\mathrm{~L}^{3} / \mathrm{L}^{3}\right]$. The porous domain 1 is continuously connected and much more conductive and diffusive than the porous domain 2: $K_{2} / K_{1}=O\left(\varepsilon^{2}\right)$ and $D_{2} / D_{1}=O\left(\varepsilon^{2}\right)$, where subscripts " 1 " and " 2 " denote the domains 1 and 2, respectively; $\boldsymbol{K}_{1}\left(\theta_{1}\right)\left[\mathrm{LT}^{-1}\right]$ and $\boldsymbol{K}_{2}\left(\theta_{2}\right)\left[\mathrm{LT}^{-1}\right]$ are the hydraulic conductivity tensors, and $\boldsymbol{D}_{1}\left(\theta_{1}\right)\left[\mathrm{L}^{2} \mathrm{~T}^{-1}\right]$ and $\boldsymbol{D}_{2}\left(\theta_{2}\right)\left[\mathrm{L}^{2} \mathrm{~T}^{-1}\right]$ are the effective diffusion tensors. Therefore, the effects of preferential flow/transport can be expected. The strong ratio $D_{2} / D_{1}=O\left(\varepsilon^{2}\right)$ can be referred to a situation of solute diffusion in polycrystals (Brassart and Stainier 2019) or helium diffusing in silica (silicon dioxide) inclusions embedded in a coarse sand, for example. This strong contrast may also be reached when hindered diffusion occurs in the porous domain 2 (i.e. when the diffusing molecules are quite large with respect to the pore size).

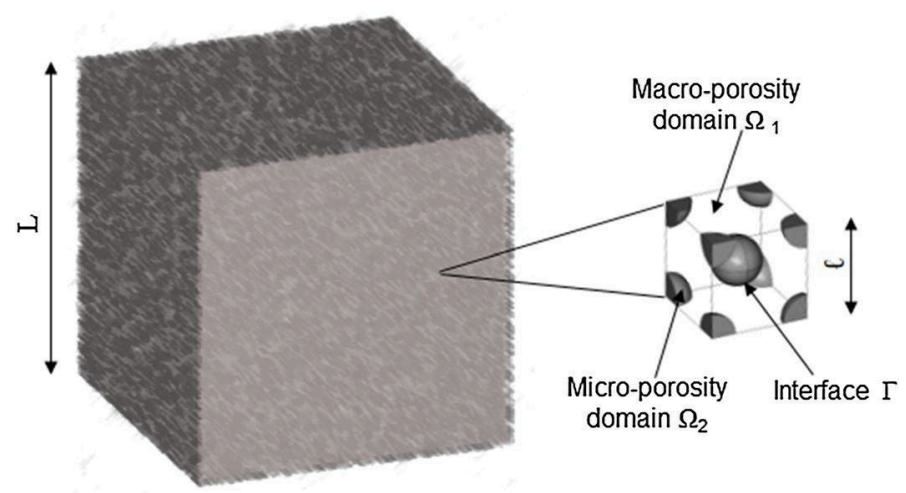

Fig. 1 Periodic double-porosity medium with the macro- and microscopic characteristic lengths and the microstructure with two porous ingredients 
The double-porosity medium is a double-structured medium with three distinct different scales: the pore scale, the Darcy scale and the macroscopic scale. In this study, the starting point of the analysis is the Darcy scale (hereinafter named microscopic scale or local scale).

\subsection{Transport Description at the Microscopic Scale}

At the $\ell$ scale, we assume that under the unsaturated steady-state flow the transport of a nonreactive solute can be described in each porous domain by the mass conservation equation (Bear 1972):

$$
\begin{aligned}
& \frac{\partial\left(\theta_{1} C_{1}\right)}{\partial t}=\nabla_{X} \cdot\left(\boldsymbol{D}_{1}\left(\theta_{1}\right) \cdot \nabla_{X} C_{1}-v_{1} C_{1}\right) \quad \text { in } \Omega_{1} \\
& \frac{\partial\left(\theta_{2} C_{2}\right)}{\partial t}=\nabla_{X} \cdot\left(\boldsymbol{D}_{2}\left(\theta_{2}\right) \cdot \nabla_{X} C_{2}-v_{2} C_{2}\right) \quad \text { in } \Omega_{2}
\end{aligned}
$$

together with the continuity conditions of the flux and the concentrations at the interface:

$$
\begin{aligned}
\left(\boldsymbol{D}_{1}\left(\theta_{1}\right) \cdot \nabla_{X} C_{1}-\boldsymbol{v}_{1} C_{1}\right) \cdot \boldsymbol{N} & =\left(\boldsymbol{D}_{2}\left(\theta_{2}\right) \cdot \nabla_{X} C_{2}-\boldsymbol{v}_{2} C_{2}\right) \cdot \boldsymbol{N} \text { on } \Gamma \\
C_{1} & =C_{2} \quad \text { on } \Gamma
\end{aligned}
$$

where $C_{1}\left[\mathrm{ML}^{-3}\right]$ and $C_{2}\left[\mathrm{ML}^{-3}\right]$ are the solute concentrations; the $X=\left(X_{1}, X_{2}, X_{3}\right)[\mathrm{L}]$ denotes the physical spatial variable with $X_{3}$ oriented positively upwards; $t$ [T] is the time; $v_{1}$ and $v_{2}\left[\mathrm{LT}^{-1}\right]$ are the local Darcy velocities; and $N[-]$ is the unit vector normal to $\Gamma$ and exterior to $\Omega_{1}$.

The unsaturated water flow in each homogeneous rigid domain is described by the Richards equation (Richards 1931). We assume that the flow fields of water, considered incompressible and Newtonian, are known and determined independently from the transport problem and that the air pressure is constant and equal to the atmospheric pressure during the whole flow process. We recall the macroscopic flow equation in the double-porosity medium obtained by Lewandowska et al. (2004) using the homogenization approach:

$$
\phi_{1} \frac{\partial \theta_{1}}{\partial t}=\nabla_{X} \cdot\left[\boldsymbol{K}_{\mathrm{eff}}(h) \cdot \nabla_{X}\left(h+X_{3}\right)\right]-W(h, t)
$$

where $\boldsymbol{K}_{\mathrm{eff}}\left[\mathrm{LT}^{-1}\right]$ is the effective hydraulic conductivity depending on the conductivity of the porous domain $\Omega_{1}$ and the geometry of the microstructure; $h[\mathrm{~L}]$ is the water pressure head; $\phi_{1}[-]$ is the volumetric fraction of the macro-porosity domain 1, defined by $\phi_{1}=\left|\Omega_{1}\right| /|\Omega|$ and is of the order $O(1)$; and $W(h, t)\left[\mathrm{T}^{-1}\right]$ is the source term of water exchange between the two domains. Under the steady-state condition, Eq. (6) becomes

$$
\nabla_{X} \cdot\left[\boldsymbol{K}_{\mathrm{eff}}(h) \cdot \nabla_{X}\left(h+X_{3}\right)\right]=0
$$

with

$$
\langle\boldsymbol{v}\rangle=\left\langle\boldsymbol{v}_{1}\right\rangle=\left[\boldsymbol{K}_{\mathrm{eff}}(h) \cdot \nabla_{X}\left(h+X_{3}\right)\right]
$$


defining the macroscopic velocity $\left[\mathrm{LT}^{-1}\right]$. Moreover, according to the result of the flow problem in the double-porosity medium obtained by homogenization, the flow only occurs in the domain 1 (macro-porosity domain) and water is stagnant in the domain 2 (microporosity domain) (see detail in Lewandowska et al. 2004), we have:

$$
\begin{aligned}
& \nabla_{y} \cdot \boldsymbol{v}_{1}^{(0)}=0 \quad \text { in } \Omega_{1} \\
& \boldsymbol{v}_{1}^{(0)} \cdot \boldsymbol{N}=0 \quad \text { on } \Gamma .
\end{aligned}
$$

The problem (2)-(5) can be rewritten as:

$$
\begin{gathered}
\frac{\partial\left(\theta_{1} C_{1}\right)}{\partial t}=\nabla_{X} \cdot\left(\boldsymbol{D}_{1}\left(\theta_{1}\right) \cdot \nabla_{X} C_{1}-\boldsymbol{v}_{1} C_{1}\right) \quad \text { in } \Omega_{1} \\
\frac{\partial\left(\theta_{2} C_{2}\right)}{\partial t}=\nabla_{X} \cdot\left(\boldsymbol{D}_{2}\left(\theta_{2}\right) \cdot \nabla_{X} C_{2}\right) \quad \text { in } \Omega_{2} \\
\left(\boldsymbol{D}_{1}\left(\theta_{1}\right) \cdot \nabla_{X} C_{1}-v_{1} C_{1}\right) \cdot \boldsymbol{N}=\left(\boldsymbol{D}_{2}\left(\theta_{2}\right) \cdot \nabla_{X} C_{2}\right) \cdot \boldsymbol{N} \text { on } \Gamma \\
C_{1}=C_{2} \quad \text { on } \Gamma .
\end{gathered}
$$

\subsection{Dimensionless Microscopic Model with Estimated Characteristic Numbers}

Let us normalize the variables with respect to their characteristic values denoted herein by the subscript " $c$ ". One leads to the following dimensionless variables denoted by the superscript "**":

$$
C_{1}^{*}=\frac{C_{1}}{C_{1 c}} ; \quad C_{2}^{*}=\frac{C_{2}}{C_{2 c}} ; \quad D_{1}^{*}=\frac{\boldsymbol{D}_{1}}{D_{1 c}} ; \quad \boldsymbol{D}_{2}^{*}=\frac{\boldsymbol{D}_{2}}{D_{2 c}} ; \quad \theta_{1}^{*}=\frac{\theta_{1}}{\theta_{1 c}} ; \quad \theta_{2}^{*}=\frac{\theta_{2}}{\theta_{2 c}} ; \quad v_{1}^{*}=\frac{v_{1}}{v_{1 c}} ; \quad t^{*}=\frac{t}{t_{c}} .
$$

We divide also the spatial variable by the microscopic and macroscopic characteristic length, respectively, to obtain two dimensionless space variables:

$$
\boldsymbol{y}=\frac{X}{\ell} \quad \text { and } \quad x=\frac{X}{L}
$$

where $y\left(y_{1}, y_{2}, y_{3}\right)$ and $x\left(x_{1}, x_{2}, x_{3}\right)$ are the microscopic and macroscopic dimensionless variables, respectively. From the microscopic point of view, the local problem (11)-(14) normalized with respect to $\ell$ in terms of dimensionless unknowns (15) and (16) becomes:

$$
\begin{gathered}
\frac{\ell^{2}}{D_{1 c} t_{c}} \frac{\partial\left(\theta_{1}^{*} C_{1}^{*}\right)}{\partial t^{*}}=\nabla_{y} \cdot\left(\boldsymbol{D}_{1}^{*} \cdot \nabla_{y} C_{1}^{*}-\frac{\ell v_{1 c}}{D_{1 c}} \boldsymbol{v}_{1}^{*} C_{1}^{*}\right) \quad \text { in } \Omega_{1} \\
\frac{\ell^{2}}{D_{2 c} t_{c}} \frac{\partial\left(\theta_{2}^{*} C_{2}^{*}\right)}{\partial t^{*}}=\nabla_{y} \cdot\left(\boldsymbol{D}_{2}^{*} \cdot \nabla_{y} C_{2}^{*}\right) \quad \text { in } \Omega_{2}
\end{gathered}
$$




$$
\begin{gathered}
\left(\boldsymbol{D}_{1}^{*} \cdot \nabla_{y} C_{1}^{*}-\frac{\ell v_{1 c}}{D_{1 c}} v_{1}^{*} C_{1}^{*}\right) \cdot N=\frac{D_{2 c} C_{2 c}}{D_{1 c} C_{1 c}}\left(D_{2}^{*} \cdot \nabla_{y} C_{2}^{*}\right) \cdot N \text { on } \Gamma \\
C_{1 c} C_{1}^{*}=C_{2 c} C_{2}^{*} \quad \text { on } \Gamma .
\end{gathered}
$$

In the dimensionless microscopic problem (17)-(20), we identify the following dimensionless quantities:

$$
P e_{1 \ell}=\frac{\ell v_{1 c}}{D_{1 c}} \text { (Péclet number); } P_{1 \ell}=\frac{\ell^{2}}{D_{1 c} t_{c}} ; P_{2 \ell}=\frac{\ell^{2}}{D_{2 c} t_{c}} \text { and } Q=\frac{D_{2 c}}{D_{1 c}} \text {. }
$$

We establish the order of magnitude of the above dimensionless numbers in power of $\varepsilon$ in a manner involving the dispersion phenomena, the double-porosity behaviours and the resulting local non-equilibrium. To do that, the Péclet number is chosen to consider the equivalence of the convective and diffusive effects at the micro-scale such that $P e_{1 \ell}=O(1)$ and the ratio $Q=O\left(\varepsilon^{2}\right)$. The latter can be named the local diffusion case which is different from the local dispersion case presented in Tran Ngoc et al. (2011). The characteristic time, chosen as the reference diffusion time $t_{c}=L^{2} / D_{1 c}$ over $L$ (time of the observation), is postulated as well as the ratio $Q$. Finally, we assume $C_{1 c}$ to be of the same order of magnitude as $C_{2 c}$. Thus, we will proceed the homogenization of the dimensionless problem (17)-(20) with the estimations as follows:

$$
\begin{gathered}
t_{c}=\frac{L^{2}}{D_{1 c}} ; P e_{1 \ell}=\frac{\ell v_{1 c}}{D_{1 c}}=O(1) ; Q=\frac{D_{2 c}}{D_{1 c}}=O\left(\varepsilon^{2}\right) \text { so that } \\
P_{1 \ell}=\frac{\ell^{2}}{D_{1 c} t_{c}}=O\left(\varepsilon^{2}\right) \text { and } P_{2 \ell}=\frac{\ell^{2}}{D_{2 c} t_{c}}=O(1) .
\end{gathered}
$$

Making use of the estimates (22), we get

$$
\begin{gathered}
\varepsilon^{2} \frac{\partial\left(\theta_{1}^{*} C_{1}^{*}\right)}{\partial t^{*}}=\nabla_{y} \cdot\left(\boldsymbol{D}_{1}^{*} \cdot \nabla_{y} C_{1}^{*}-\boldsymbol{v}_{1}^{*} C_{1}^{*}\right) \quad \text { in } \Omega_{1} \\
\frac{\partial\left(\theta_{2}^{*} C_{2}^{*}\right)}{\partial t^{*}}=\nabla_{y} \cdot\left(\boldsymbol{D}_{2}^{*} \cdot \nabla_{y} C_{2}^{*}\right) \quad \text { in } \Omega_{2} \\
\left(\boldsymbol{D}_{1}^{*} \cdot \nabla_{y} C_{1}^{*}-v_{1}^{*} C_{1}^{*}\right) \cdot \boldsymbol{N}=\varepsilon^{2}\left(\boldsymbol{D}_{2}^{*} \cdot \nabla_{y} C_{2}^{*}\right) \cdot \boldsymbol{N} \text { on } \Gamma \\
C_{1}^{*}=C_{2}^{*} \quad \text { on } \Gamma .
\end{gathered}
$$

\section{Modelling by Homogenization}

We applied here the procedure of homogenization by two-scale asymptotic expansions for the engineering problems pioneered by Auriault (1983, 1991). Homogenization postulates that all the unknowns $\psi$ can be expressed in the form of asymptotic expansions in powers of $\varepsilon$

$$
\psi\left(\boldsymbol{x}, \boldsymbol{y}, t^{*}\right)=\psi^{(0)}\left(\boldsymbol{x}, \boldsymbol{y}, t^{*}\right)+\varepsilon \psi^{(1)}\left(\boldsymbol{x}, \boldsymbol{y}, t^{*}\right)+\varepsilon^{2} \psi^{(2)}\left(\boldsymbol{x}, \boldsymbol{y}, t^{*}\right)+\cdots
$$


where $\psi\left(\boldsymbol{x}, \boldsymbol{y}, t^{*}\right)$ stands for $C_{1}^{*}, C_{2}^{*}$ or $\boldsymbol{v}_{1}^{*}$ and is spatially periodic in $\boldsymbol{y}$ over both subdomains. All the terms $\psi^{(\mathrm{i})}\left(\boldsymbol{x}, \boldsymbol{y}, t^{*}\right)$ in Eq. (27) are dimensionless without the ${ }^{*}$ superscript, in order to simplify the notation. Due to (16) and (1), we have the relation $\boldsymbol{x}=\varepsilon \boldsymbol{y}$ that allows the derivation operator to be transformed into

$$
\nabla_{y} \mapsto \nabla_{y}+\varepsilon \nabla_{x}
$$

Applying (27) and (28) to the microscopic model Eqs. (23)-(26), regrouping the terms at the same order of $\varepsilon^{0}-\varepsilon^{2}$ and neglecting the terms with a higher order of $\varepsilon^{2}$ leads to:

$$
\begin{aligned}
& \varepsilon^{2} \frac{\partial\left(\theta_{1}^{*} C_{1}^{(0)}\right)}{\partial t^{*}}+\cdots=\nabla_{y} \cdot\left[\boldsymbol{D}_{1}^{*} \cdot \nabla_{y} C_{1}^{(0)}-\boldsymbol{v}_{1}^{(0)} C_{1}^{(0)}\right] \\
& +\varepsilon\left[\begin{array}{r}
\nabla_{y} \cdot\left(D_{1}^{*} \cdot \nabla_{y} C_{1}^{(1)}+D_{1}^{*} \cdot \nabla_{x} C_{1}^{(0)}\right)-\nabla_{y} \cdot\left(v_{1}^{(0)} C_{1}^{(1)}+v_{1}^{(1)} C_{1}^{(0)}\right) \\
-\nabla_{x} \cdot\left(D_{1}^{*} \cdot \nabla_{y} C_{1}^{(0)}\right)-\nabla_{x} \cdot\left(v_{1}^{(0)} C_{1}^{(0)}\right)
\end{array}\right] \\
& +\varepsilon^{2}\left[\begin{array}{r}
\nabla_{y} \cdot\left(\boldsymbol{D}_{1}^{*} \cdot \nabla_{y} C_{1}^{(2)}+\boldsymbol{D}_{1}^{*} \cdot \nabla_{x} C_{1}^{(1)}\right)-\nabla_{y} \cdot\left(\boldsymbol{v}_{1}^{(0)} C_{1}^{(2)}+\boldsymbol{v}_{1}^{(1)} C_{1}^{(1)}+\boldsymbol{v}_{1}^{(2)} C_{1}^{(0)}\right) \\
+\nabla_{x} \cdot\left(\boldsymbol{D}_{1}^{*} \cdot \nabla_{y} C_{1}^{(1)}+\boldsymbol{D}_{1}^{*} \cdot \nabla_{x} C_{1}^{(0)}\right) \\
-\nabla_{x} \cdot\left(\boldsymbol{v}_{1}^{(0)} C_{1}^{(1)}+\boldsymbol{v}_{1}^{(1)} C_{1}^{(0)}\right)
\end{array}\right] \\
& +\cdots \quad \text { in } \Omega_{1} \\
& \frac{\partial\left(\theta_{2}^{*} C_{2}^{(0)}\right)}{\partial t^{*}}+\varepsilon \frac{\partial\left(\theta_{2}^{*} C_{2}^{(1)}\right)}{\partial t^{*}}+\varepsilon^{2} \frac{\partial\left(\theta_{2}^{*} C_{2}^{(2)}\right)}{\partial t^{*}}+\cdots=\nabla_{y} \cdot\left(\boldsymbol{D}_{2}^{*} \cdot \nabla_{y} C_{2}^{(0)}\right) \\
& +\varepsilon\left[\nabla_{y} \cdot\left(\boldsymbol{D}_{2}^{*} \cdot \nabla_{y} C_{2}^{(1)}+\boldsymbol{D}_{2}^{*} \cdot \nabla_{x} C_{2}^{(0)}\right)+\nabla_{x} \cdot\left(\boldsymbol{D}_{2}^{*} \cdot \nabla_{y} C_{2}^{(0)}\right)\right] \\
& +\varepsilon^{2}\left[\nabla_{y} \cdot\left(\boldsymbol{D}_{2}^{*} \cdot \nabla_{y} C_{2}^{(2)}+\boldsymbol{D}_{2}^{*} \cdot \nabla_{x} C_{2}^{(1)}\right)+\nabla_{x} \cdot\left(\boldsymbol{D}_{2}^{*} \cdot \nabla_{y} C_{2}^{(1)}+\boldsymbol{D}_{2}^{*} \cdot \nabla_{x} C_{2}^{(0)}\right)\right]+\cdots \quad \text { in } \Omega_{2} \\
& \left(\boldsymbol{D}_{1}^{*} \cdot \nabla_{y} C_{1}^{(0)}-v_{1}^{(0)} C_{1}^{(0)}\right) \cdot \boldsymbol{N}+\varepsilon\left[\left(\boldsymbol{D}_{1}^{*} \cdot \nabla_{y} C_{1}^{(1)}+\boldsymbol{D}_{1}^{*} \cdot \nabla_{x} C_{1}^{(0)}-v_{1}^{(0)} C_{1}^{(1)}-v_{1}^{(1)} C_{1}^{(0)}\right) \cdot \boldsymbol{N}\right] \\
& +\varepsilon^{2}\left[\left(D_{1}^{*} \cdot \nabla_{y} C_{1}^{(2)}+D_{1}^{*} \cdot \nabla_{x} C_{1}^{(0)}-v_{1}^{(0)} C_{1}^{(2)}-v_{1}^{(1)} C_{1}^{(1)}-v_{1}^{(2)} C_{1}^{(0)}\right) \cdot N\right] \\
& +\cdots=\varepsilon^{2}\left[\left(D_{2}^{*} \cdot \nabla_{y} C_{2}^{(0)}\right) \cdot \boldsymbol{N}\right]+\cdots \quad \text { on } \Gamma \\
& \left(C_{1}^{(0)}+\varepsilon C_{1}^{(1)}+\varepsilon^{2} C_{1}^{(2)}+\cdots\right)=\left(C_{2}^{(0)}+\varepsilon C_{2}^{(1)}+\varepsilon^{2} C_{2}^{(2)}+\cdots\right) \text { on } \Gamma \text {. }
\end{aligned}
$$

Equating the terms of the same order of $\varepsilon$ in Eqs. (29)-(32) yields successive boundary value problems to be solved on the period. Hereinafter, we present only the analysis of the results of these problems at the successive orders of $\varepsilon$ allowing to obtain the effective parameters and the form of the macroscopic model.

\subsection{Macroscopic Variable}

At the order $\varepsilon^{0}$, Eqs. (29) and (31) are rewritten as 


$$
\begin{aligned}
& \nabla_{y} \cdot\left(\boldsymbol{D}_{1}^{*} \cdot \nabla_{y} C_{1}^{(0)}-v_{1}^{(0)} C_{1}^{(0)}\right)=0 \quad \text { in } \Omega_{1} \\
& \left(\boldsymbol{D}_{1}^{*} \cdot \nabla_{y} C_{1}^{(0)}-\boldsymbol{v}_{1}^{(0)} C_{1}^{(0)}\right) \cdot \boldsymbol{N}=0 \quad \text { on } \Gamma
\end{aligned}
$$

where $C_{1}^{(0)}$ is $\boldsymbol{y}$-periodic. Taking into account Eqs. (9) and (10), it can be shown that the solution of the problem (33) and (34) is (Bensoussan et al. 1978; Sanchez-Palencia 1980; Auriault and Adler 1995; Auriault and Lewandowska 1996)

$$
C_{1}^{(0)}=C_{1}^{(0)}\left(\boldsymbol{x}, t^{*}\right)=C^{(0)}\left(\boldsymbol{x}, t^{*}\right) .
$$

This means that $C_{1}^{(0)}$ is the macroscopic variable, independent of the local space variable $y$ and denoted $C^{(0)}$.

\subsection{Transport Equation in the Micro-Porosity Domain}

From Eqs. (30) and (32) at the order $\varepsilon^{0}$, we obtain the boundary value problem for the $C_{2}^{(0)}$ :

$$
\begin{gathered}
\frac{\partial\left(\theta_{2}^{(0)} C_{2}^{(0)}\right)}{\partial t^{*}}=\nabla \cdot\left(\boldsymbol{D}_{2}^{*} \cdot \nabla_{y} C_{2}^{(0)}\right) \quad \text { in } \Omega_{2} \\
C_{1}^{(0)}=C_{2}^{(0)} \quad \text { on } \Gamma .
\end{gathered}
$$

It can be seen that the problem (36) is a solute diffusion problem in the micro-porosity domain and its concentration variable depends on $\boldsymbol{y}$. Thus, the local non-equilibrium may be observed by two concentration fields $C_{1}^{(0)}\left(\boldsymbol{x}, t^{*}\right)$ and $C_{2}^{(0)}\left(\boldsymbol{x}, \boldsymbol{y}, t^{*}\right)$ balancing at the coupling boundary with the condition given by Eq. (37).

\subsection{Local Boundary Value Problem}

Let us analyse Eqs. (29) and (31) at the order $\varepsilon^{1}$ taking into account Eq. (35):

$$
\begin{gathered}
\nabla_{y} \cdot\left(D_{1}^{*} \cdot \nabla_{y} C_{1}^{(1)}+D_{1}^{*} \cdot \nabla_{x} C_{1}^{(0)}\right)-\nabla_{y} \cdot\left(v_{1}^{(0)} C_{1}^{(1)}+v_{1}^{(1)} C_{1}^{(0)}\right)-\nabla_{x} \cdot\left(v_{1}^{(0)} C_{1}^{(0)}\right)=0 \text { in } \Omega_{1} \\
\left(D_{1}^{*} \cdot \nabla_{y} C_{1}^{(1)}+D_{1}^{*} \cdot \nabla_{x} C_{1}^{(0)}-v_{1}^{(0)} C_{1}^{(1)}-v_{1}^{(1)} C_{1}^{(0)}\right) \cdot N=0 \quad \text { on } \Gamma .
\end{gathered}
$$

Integrating Eq. (38) over $\Omega_{1}$ and dividing it by $|\Omega|$, then applying the Gauss-Ostrogradsky theorem for the first term in $1 . \mathrm{h}$. s. of this equation to transform the volume integral to a surface integral and using the periodicity condition and the boundary condition given by Eq. (39) leads to

$$
\frac{1}{|\Omega|} \int_{\Omega_{1}} \nabla_{x} \cdot\left(v_{1}^{(0)} C_{1}^{(0)}\right) \mathrm{d} \Omega=0 \quad \text { or } \quad\left\langle\nabla_{x} \cdot\left(v_{1}^{(0)} C_{1}^{(0)}\right)\right\rangle=\nabla_{x} \cdot\left(\left\langle v_{1}^{(0)}\right\rangle C_{1}^{(0)}\right)=0 .
$$

here the intrinsic volume average is defined by 


$$
\langle\cdot\rangle=\frac{1}{|\Omega|} \int_{\Omega_{1}} \cdot \mathrm{d} \Omega .
$$

The problem given by Eqs. (38) and (39) defines $C_{1}^{(1)}$ and the solution can be put in the form of a linear function of $\nabla_{x} C_{1}^{(0)}$ (Bensoussan et al. 1978; Sanchez-Palencia 1980; Auriault and Adler 1995; Auriault and Lewandowska 1996)

$$
C_{1}^{(1)}=\chi(\boldsymbol{y}) \cdot \nabla_{x} C_{1}^{(0)}+\bar{C}_{1}^{(1)}\left(\boldsymbol{x}, t^{*}\right)
$$

where $\chi(y)$ is $\boldsymbol{y}$-periodic and considered as a corrector of the gradient of $C_{1}^{(0)}$ with respect to $x$ reflecting the influence of the microstructural geometry. This function satisfies the zerovalued volume average condition for uniqueness:

$$
\langle\chi(y)\rangle=0
$$

$\overline{\boldsymbol{C}}_{1}^{(1)}\left(\boldsymbol{x}, t^{*}\right)$ is an arbitrary function independent of $\boldsymbol{y}$, therefore a macroscopic concentration satisfying $\nabla_{y} \bar{C}_{1}^{(1)}\left(\boldsymbol{x}, t^{*}\right)=0$

In order to obtain the local problem allowing to calculate the vector $\chi(y)$, we introduce Eq. (42) into Eq. (38) and analyse it term by term. The first term, on the 1. h. s. becomes

$$
\nabla_{y} \cdot\left(\boldsymbol{D}_{1}^{*} \cdot \nabla_{y} C_{1}^{(1)}+\boldsymbol{D}_{1}^{*} \cdot \nabla_{x} C_{1}^{(0)}\right)=\nabla_{y} \cdot\left[\boldsymbol{D}_{1}^{*} \cdot\left(\nabla_{y} \chi+\boldsymbol{I}\right)\right] \nabla_{x} C_{1}^{(0)}
$$

where $\boldsymbol{I}$ is the identity matrix. The second term can be transformed as

$$
\nabla_{y} \cdot\left(v_{1}^{(0)} C_{1}^{(1)}+v_{1}^{(1)} C_{1}^{(0)}\right)=C_{1}^{(1)} \nabla_{y} \cdot v_{1}^{(0)}+v_{1}^{(0)} \cdot \nabla_{y} C_{1}^{(1)}+C_{1}^{(0)} \nabla_{y} \cdot v_{1}^{(1)}+v_{1}^{(1)} \cdot \nabla_{y} C_{1}^{(0)}
$$

We have had $\nabla_{y} \cdot v_{1}^{(0)}=0$ (Eq. 9) and $C_{1}^{(0)}$ does not depend on $\boldsymbol{y}$, so Eq. (45) becomes

$$
\nabla_{y} \cdot\left(v_{1}^{(0)} C_{1}^{(1)}+v_{1}^{(1)} C_{1}^{(0)}\right)=v_{1}^{(0)} \cdot \nabla_{y} \cdot\left(\chi(y) \nabla_{x} C_{1}^{(0)}\right)+C_{1}^{(0)} \nabla_{y} \cdot v_{1}^{(1)}
$$

The third term gives

$$
\nabla_{x} \cdot\left(\mathrm{v}_{1}^{(0)} C_{1}^{(0)}\right)=C_{1}^{(0)} \nabla_{x} \cdot \mathrm{v}_{1}^{(0)}+\mathrm{v}_{1}^{(0)} \cdot \nabla_{x} C_{1}^{(0)}
$$

Using the above obtained results, Eq. (40) is reformulated in the following form

$$
\begin{aligned}
\nabla_{y} \cdot & {\left[\boldsymbol{D}_{1}^{*} \cdot\left(\nabla_{y} \chi+\boldsymbol{I}\right)\right] \nabla_{x} C_{1}^{(0)}-\boldsymbol{v}_{1}^{(0)} \cdot \nabla_{y} \cdot\left(\chi \nabla_{x} C_{1}^{(0)}\right) } \\
& -C_{1}^{(0)} \nabla_{y} \cdot \boldsymbol{v}_{1}^{(1)}-C_{1}^{(0)} \nabla_{x} \cdot \boldsymbol{v}_{1}^{(0)}-\boldsymbol{v}_{1}^{(0)} \cdot \nabla_{x} C_{1}^{(0)}=0 \text { in } \Omega_{1} .
\end{aligned}
$$

Let us now analyse the sum of third and fourth terms in 1. h. s. of Eq. (48)

$$
C_{1}^{(0)} \nabla_{y} \cdot \boldsymbol{v}_{1}^{(1)}+C_{1}^{(0)} \nabla_{x} \cdot \boldsymbol{v}_{1}^{(0)}=C_{1}^{(0)}\left(\nabla_{y} \cdot \boldsymbol{v}_{1}^{(1)}+\nabla_{x} \cdot \boldsymbol{v}_{1}^{(0)}\right)
$$

Due to the flow problem at the order $\varepsilon^{2}$, Eq. (49) can be written as

$$
C_{1}^{(0)}\left(\nabla_{y} \cdot v_{1}^{(1)}+\nabla_{x} \cdot v_{1}^{(0)}\right)=C_{1}^{(0)} \frac{\partial \theta_{1}^{(0)}}{\partial t^{*}} .
$$


On the other hand, Eq. (6) can be rewritten in the dimensionless form

$$
\phi_{1} \frac{\partial \theta_{1}^{(0)}}{\partial t^{*}}=\nabla_{x} \cdot\left\langle v_{1}^{(0)}\right\rangle-W\left(h^{(0)}, t^{*}\right)
$$

hence we obtain for Eq. (50)

$$
C_{1}^{(0)}\left(\nabla_{y} \cdot v_{1}^{(1)}+\nabla_{x} \cdot v_{1}^{(0)}\right)=C_{1}^{(0)}\left[\frac{1}{\phi_{1}} \nabla_{x} \cdot\left\langle v_{1}^{(0)}\right\rangle-\frac{1}{\phi_{1}} W\left(h^{(0)}, t^{*}\right)\right] .
$$

Now, returning to Eq. (40), its development gives

$$
\nabla_{x} \cdot\left(\left\langle v_{1}^{(0)}\right\rangle C_{1}^{(0)}\right)=C_{1}^{(0)} \nabla_{x} \cdot\left\langle v_{1}^{(0)}\right\rangle+\left\langle v_{1}^{(0)}\right\rangle \cdot \nabla_{x} C_{1}^{(0)}=0
$$

or

$$
C_{1}^{(0)} \nabla_{x} \cdot\left\langle v_{1}^{(0)}\right\rangle=-\left\langle v_{1}^{(0)}\right\rangle \cdot \nabla_{x} C_{1}^{(0)}
$$

With the result of the replacement (54) into (52) and the fact that the water exchange term $W\left(h^{(0)}, t^{*}\right)$ disappears from the development due to the steady-state flow condition, Eq. (48) becomes

$$
\begin{gathered}
\nabla_{y} \cdot\left[\boldsymbol{D}_{1}^{*} \cdot\left(\nabla_{y} \chi+\boldsymbol{I}\right)\right] \nabla_{x} C_{1}^{(0)}-\boldsymbol{v}_{1}^{(0)} \cdot \nabla_{y} \cdot\left(\chi \nabla_{x} C_{1}^{(0)}\right) \\
+\frac{1}{\phi_{1}}\left\langle\boldsymbol{v}_{1}^{(0)}\right\rangle \cdot \nabla_{x} C_{1}^{(0)}-\boldsymbol{v}_{1}^{(0)} \cdot \nabla_{x} C_{1}^{(0)}=0 \quad \text { in } \Omega_{1} .
\end{gathered}
$$

Taking into account the boundary condition on $\Gamma$ for steady-state water flow, Eq. (39) is reduced to

$$
\left(\boldsymbol{D}_{1}^{*} \nabla_{y} C_{1}^{(1)}+\boldsymbol{D}_{1}^{*} \nabla_{x} C_{1}^{(0)}\right) \cdot \boldsymbol{N}=0 \quad \text { on } \Gamma
$$

and then replacing $C_{1}^{(1)}$ by the expression given in Eq. (42) leads to

$$
\left[\boldsymbol{D}_{1}^{*} \cdot\left(\nabla_{y} \chi+\boldsymbol{I}\right) \nabla_{x} C_{1}^{(0)}\right] \cdot \boldsymbol{N}=0 \quad \text { on } \Gamma .
$$

Imposing successively the unit gradient $\nabla_{x} C_{1}^{(0)}$ in the directions 1,2 and 3 , we obtain the local problem for $\chi(y)$

$$
\begin{gathered}
\nabla_{y} \cdot\left[\boldsymbol{D}_{1}^{*} \cdot\left(\nabla_{y} \chi+\boldsymbol{I}\right)\right]-\boldsymbol{v}_{1}^{(0)} \cdot \nabla_{y} \chi=\boldsymbol{v}_{1}^{(0)}-\frac{1}{\phi_{1}}\left\langle\boldsymbol{v}_{1}^{(0)}\right\rangle \quad \text { in } \Omega_{1} \\
{\left[\boldsymbol{D}_{1}^{*} \cdot\left(\nabla_{y} \chi+\boldsymbol{I}\right)\right] \cdot \boldsymbol{N}=0 \quad \text { on } \Gamma}
\end{gathered}
$$

and we recall the condition by Eq. (43) which assures the unicity of the solution.

This local boundary value problem, Eqs. (58)-(59), has the same form as the one obtained by Auriault and Adler (1995) and Auriault and Lewandowska (1996) for the saturated conditions. 


\subsection{Macroscopic Model}

In this section, we present the development of the macroscopic model by analysing the homogenization of the problem given by Eqs. (29) and (31) at the order $O\left(\varepsilon^{2}\right)$ of approximation

$$
\begin{aligned}
& \frac{\partial\left(\theta_{1}^{*} C_{1}^{(0)}\right)}{\partial t^{*}}=\nabla_{y} \cdot\left(D_{1}^{*} \cdot \nabla_{y} C_{1}^{(2)}+D_{1}^{*} \cdot \nabla_{x} C_{1}^{(1)}\right)-\nabla_{y} \cdot\left(v_{1}^{(0)} C_{1}^{(2)}+v_{1}^{(1)} C_{1}^{(1)}+v_{1}^{(2)} C_{1}^{(0)}\right) \\
& +\nabla_{x} \cdot\left(D_{1}^{*} \cdot \nabla_{y} C_{1}^{(1)}+D_{1}^{*} \cdot \nabla_{x} C_{1}^{(0)}\right)-\nabla_{x} \cdot\left(v_{1}^{(0)} C_{1}^{(1)}+v_{1}^{(1)} C_{1}^{(0)}\right) \quad \text { in } \Omega_{1} \\
& \left(D_{1}^{*} \cdot \nabla_{y} C_{1}^{(2)}+D_{1}^{*} \cdot \nabla_{x} C_{1}^{(0)}-v_{1}^{(0)} C_{1}^{(2)}-v_{1}^{(1)} C_{1}^{(1)}-v_{1}^{(2)} C_{1}^{(0)}\right) \cdot \boldsymbol{N}=\left(D_{2}^{*} \cdot \nabla_{y} C_{2}^{(0)}\right) \cdot \boldsymbol{N} \quad \text { on } \Gamma \text {. }
\end{aligned}
$$

The following transformations are successively conducted: (1) integrating Eq. (60) over $\Omega_{1}$ and dividing it by $|\Omega|$; (2) applying the Gauss-Ostrogradsky theorem for the first two terms in r. h. s. of Eq. (60); (3) applying the periodicity condition and the boundary condition Eq. (61). After these transformations, we obtain

$$
\begin{aligned}
\frac{\partial\left\langle\theta_{1}^{*} C_{1}^{(0)}\right\rangle}{\partial t^{*}}= & \frac{1}{|\Omega|} \int_{\Gamma}\left(\boldsymbol{D}_{2}^{*} \cdot \nabla_{y} C_{2}^{(0)}\right) \cdot \boldsymbol{N} \mathrm{d} \Gamma+\nabla_{x} \cdot\left\langle\boldsymbol{D}_{1}^{*} \cdot \nabla_{y} C_{1}^{(1)}+\boldsymbol{D}_{1}^{*} \cdot \nabla_{x} C_{1}^{(0)}\right\rangle \\
& -\nabla_{x} \cdot\left\langle\boldsymbol{v}_{1}^{(0)} C_{1}^{(1)}\right\rangle-\nabla_{x} \cdot\left\langle\boldsymbol{v}_{1}^{(1)} C_{1}^{(0)}\right\rangle .
\end{aligned}
$$

Applying once again the Gauss-Ostrogradsky theorem for the first term in the r. h. s. of Eq. (62) to pass from the surface to the volume integral over the domain $\Omega_{2}$ and taking into account Eq. (36) leads to

$$
\begin{gathered}
\frac{1}{|\Omega|} \int_{\Gamma}\left(\boldsymbol{D}_{2}^{*} \cdot \nabla_{y} C_{2}^{(0)}\right) \cdot N \mathrm{~d} \Gamma=-\frac{1}{|\Omega|} \int_{\Omega_{2}} \nabla_{y} \cdot\left(\boldsymbol{D}_{2}^{*} \cdot \nabla_{y} C_{2}^{(0)}\right) \mathrm{d} \Omega \text { and } \\
\quad-\frac{1}{|\Omega|} \int_{\Omega_{2}} \nabla_{y} \cdot\left(\boldsymbol{D}_{2}^{*} \cdot \nabla_{y} C_{2}^{(0)}\right) \mathrm{d} \Omega=-\frac{1}{|\Omega|} \int_{\Omega_{2}} \frac{\partial\left(\theta_{2}^{*} C_{2}^{(0)}\right)}{\partial t^{*}} \mathrm{~d} \Omega .
\end{gathered}
$$

From Eqs. (63) and (64) and the replacement of the expression of $C_{1}^{(1)}$ given by Eq. (42), we can rephrase Eq. (62) as

$$
\frac{\partial\left\langle\theta_{1}^{*} C_{1}^{(0)}\right\rangle}{\partial t^{*}}=\nabla_{x} \cdot\left(\boldsymbol{D}_{\mathrm{eff}}^{*} \cdot \nabla_{x} C_{1}^{(0)}\right)-\nabla_{x} \cdot\left\langle\boldsymbol{v}_{1}^{(0)} \bar{C}_{1}^{(1)}\right\rangle-\nabla_{x} \cdot\left\langle\boldsymbol{v}_{1}^{(1)} C_{1}^{(0)}\right\rangle-\frac{\partial\left\langle\theta_{2}^{*} C_{2}^{(0)}\right\rangle}{\partial t^{*}}
$$

where

$$
\boldsymbol{D}_{\mathrm{eff}}^{*}(\theta)=\frac{1}{|\Omega|} \int_{\Omega_{1}}\left[\boldsymbol{D}_{1}^{*}(\theta) \cdot\left(\nabla_{y} \chi+\boldsymbol{I}\right)-\boldsymbol{v}_{1}^{(0)} \cdot \chi\right] \mathrm{d} \Omega
$$


In order to obtain the macroscopic equation, we perform a similar process as in Auriault and Lewandowska (1996). Equation (65) multiplied by $\varepsilon$ will be added to Eq. (40)

$$
\begin{aligned}
\varepsilon \frac{\partial\left\langle\theta_{1}^{*} C_{1}^{(0)}\right\rangle}{\partial t^{*}}= & \varepsilon \nabla_{x} \cdot\left(D_{\mathrm{eff}}^{*} \cdot \nabla_{x} C_{1}^{(0)}\right)-\varepsilon \nabla_{x} \cdot\left\langle v_{1}^{(0)} \bar{C}_{1}^{(1)}\right\rangle-\varepsilon \nabla_{x} \cdot\left\langle v_{1}^{(1)} C_{1}^{(0)}\right\rangle \\
& -\varepsilon \frac{\partial\left\langle\theta_{2}^{*} C_{2}^{(0)}\right\rangle}{\partial t^{*}}-\nabla_{x} \cdot\left(\left\langle v_{1}^{(1)}\right\rangle C_{1}^{(0)}\right) .
\end{aligned}
$$

The examination of all the convective terms in the r. h. s. of Eq. (67)

$$
\varepsilon \nabla_{x} \cdot\left(\left\langle\boldsymbol{v}_{1}^{(0)} \bar{C}_{1}^{(1)}\right\rangle\right)+\nabla_{x} \cdot\left(\left\langle\boldsymbol{v}_{1}^{(1)}\right\rangle C_{1}^{(0)}\right)+\varepsilon \nabla_{x} \cdot\left(\left\langle\boldsymbol{v}_{1}^{(1)} C_{1}^{(0)}\right\rangle\right)
$$

in regards with the following relations

$$
\begin{gathered}
\left\langle v_{1}^{*}\right\rangle=\left\langle v_{1}^{(0)}\right\rangle+\varepsilon\left\langle v_{1}^{(1)}\right\rangle+\cdots \text { and } \\
\left\langle C_{1}^{*}\right\rangle=\left\langle C_{1}^{(0)}\right\rangle+\varepsilon\left\langle C_{1}^{(1)}\right\rangle+\cdots \approx \phi_{1} C_{1}^{(0)}+\phi_{1} \varepsilon \bar{C}_{1}^{(1)}
\end{gathered}
$$

leads to

$$
\varepsilon \nabla_{x} \cdot\left(\left\langle\boldsymbol{v}_{1}^{(0)} \bar{C}_{1}^{(1)}\right\rangle\right)+\nabla_{x} \cdot\left(\left\langle\boldsymbol{v}_{1}^{(1)}\right\rangle C_{1}^{(0)}\right)+\varepsilon \nabla_{x} \cdot\left(\left\langle\boldsymbol{v}_{1}^{(1)} C_{1}^{(0)}\right\rangle\right)=\frac{1}{\phi_{1}} \nabla_{x} \cdot\left(\left\langle\boldsymbol{v}_{1}^{*}\right\rangle\left\langle C_{1}^{*}\right\rangle\right) .
$$

Inserting Eq. (71) into Eq. (67) yields the dimensionless macroscopic equation expressed in the form

$$
\varepsilon \phi_{1} \frac{\partial\left(\theta_{1}^{*} C_{1}^{*}\right)}{\partial t^{*}}=\varepsilon \nabla_{x} \cdot\left(\boldsymbol{D}_{\mathrm{eff}}^{*} \cdot \nabla_{x} C_{1}^{*}\right)-\nabla_{x} \cdot\left(\left\langle\boldsymbol{v}_{1}^{*}\right\rangle C_{1}^{*}\right)-\varepsilon \frac{\partial\left\langle\theta_{2}^{*} C_{2}^{*}\right\rangle}{\partial t^{*}}+O\left(\varepsilon^{2}\right) .
$$

This equation is of the $\varepsilon^{2}$ order of approximation. As expected, we can observe the presence of $\varepsilon$ in the dispersion term with regard to the convective term. After recalling Eq. (22), we obtain the dimensional double-porosity dispersion-convection model

$$
\phi_{1} \frac{\partial\left(\theta_{1} C_{1}\right)}{\partial t}=\nabla_{X} \cdot\left(\boldsymbol{D}_{\mathrm{eff}} \cdot \nabla_{X} C_{1}\right)-\nabla_{X} \cdot\left(\left\langle\boldsymbol{v}_{1}\right\rangle C_{1}\right)-\frac{\partial\left\langle\theta_{2} C_{2}\right\rangle}{\partial t}
$$

coupled with the microscopic diffusion equation in the micro-porosity $\Omega_{2}$

$$
\frac{\partial\left(\theta_{2} C_{2}\right)}{\partial t}=\nabla_{X} \cdot\left(\boldsymbol{D}_{2} \cdot \nabla_{X} C_{2}\right)
$$

and at the common interface $\Gamma$

$$
C_{1}=C_{2}
$$


where $\boldsymbol{D}_{\text {eff }}$ would be the dispersion tensor in the double-porosity medium when the medium velocity is enough high ( $P e$ is high enough). Otherwise $\boldsymbol{D}_{\text {eff }}$ is the diffusion tensor. It is defined by

$$
\boldsymbol{D}_{\text {eff }}(\theta)=\frac{1}{|\Omega|} \int_{\Omega_{1}}\left[\boldsymbol{D}_{1}(\theta) \cdot\left(\nabla_{y} \chi+\boldsymbol{I}\right)-\boldsymbol{v}_{1} \cdot \chi\right] \mathrm{d} \Omega .
$$

The macroscopic double-porosity model with the local diffusion [Eqs. (73)-(76)] has the same form as the one presented in Tran Ngoc et al. (2011) for the case of local dispersion consisting of two equations coupled through the interface. It is similar to two-domain models of the literature with different validity domains, for example the one presented in Royer and Boutin (2012) for the case of the diffusivity ratio $D_{2} / D_{1}=O(\varepsilon)$. Equations (75)-(76) lead to memory effects (which give rise to tailing effects in the breakthrough curves) as shown in several works cited in the paper. It describes two mutual solute transport processes namely dispersion-convection in the macro-porosity domain and diffusion in the micro-porosity domain. This situation induces a local non-equilibrium of the concentrations by the presence of the source term in Eq. (73) coming from the interaction with the micro-porosity domain. This causes particular effects in the transport phenomena which cannot be captured by classic models. Note that the effective parameter of the model is an anisotropic dispersion tensor $\boldsymbol{D}_{\text {eff }}$ given by Eq. (76) as a function of the local diffusion tensor $\boldsymbol{D}_{1}$, local velocity vector $\boldsymbol{v}_{1}$ and the function characterizing the micro-geometry of the period $\chi$.

\section{Numerical Validation}

In order to validate the double-porosity model developed in this work, we consider in this section 2D cases in which a solute is transported by a given unsaturated flow in a periodic double-porosity medium. The numerical implementation macroscopic boundary value problem [Eqs. (73)-(76)] was performed using the commercial element finite code COMSOL Multiphysics ${ }^{\circledR}$. A particular strategy enabling to compute the concentrations at two scales corresponding to the macro- and micro-porosity domains was developed. The obtained solutions of the macroscopic model will be compared with the reference solutions of the fine scale model (direct numerical simulation) in order to validate numerically the developed double-porosity model.

\subsection{Description of the Numerical Test Cases and the Fine Scale Direct Simulation}

The geometry of the double-porosity medium is represented by circular inclusions (microporous domain assumed isotropic for simplicity) embedded in the matrix (macro-porous domain, also assumed isotropic) (Fig. 2). For the numerical verification, we chose a macroscopic domain $L=2 \times 10^{-2} \mathrm{~m}$ containing 10 periods $\ell=2 \times 10^{-3} \mathrm{~m}$ (Fig. 2) (Davit and Quintard 2017). The $\ell / L$ ratio and the number of periods are comparable to the ones considered in the numerical model used to reproduce the transport experiments in Golfier et al. (2007). The macroscopic length is relatively short but acceptable for the relatively small flux imposed. Thus, the expected features of the developed model can be ensured. The diameter of the circular inclusion is equal to half of the period length, $2 R=1 \times 10^{-3}$ $\mathrm{m}$ where $R$ is the radius of the inclusion. The hydraulic and transport properties are very 


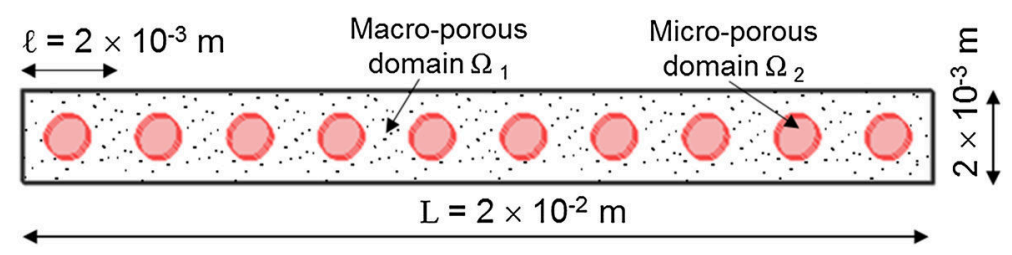

Fig. 2 Periodic double-porosity medium (10 periods) of the numerical test cases 1 and 2 . The radius of the circular inclusions (micro-porous domain 2 ) is of $0.5 \times 10^{-3} \mathrm{~m}$

Table 1 Properties of the periodic structure for the numerical examples

\begin{tabular}{lll}
\hline Geometry size $(\mathrm{m})$ & $\ell=2 \times 10^{-3} ; L=2 \times 10^{-2}$ & \\
\cline { 2 - 3 } Physical properties & Macro-porous domain 1 & Micro-porous domain 2 \\
\hline Volume fraction (-) & $\phi_{1}=0.804$ & $\phi_{2}=0.196$ \\
Water content (-) & $\theta_{1}=0.50$ & $\theta_{2}=0.30$ \\
Diffusion coefficient $\left(\mathrm{m}^{2} / \mathrm{s}\right)$ & $D_{1}\left(\theta_{1}\right)=1 \times 10^{-9}$ & $D_{2}\left(\theta_{2}\right)=1 \times 10^{-13}$ \\
\hline
\end{tabular}

contrasted between the two porous domains to respect the characteristics of double-porosity media. The physical properties of the porous media used in the two numerical examples are reported in Table 1.

For a fixed water content $\langle\theta\rangle$, we assume a given Darcy velocity $\langle v\rangle(\theta)=0.796 \times 10^{-7} \mathrm{~m} / \mathrm{s}$ at the inlet of the medium leading in conformity with the condition on the Peclet number of $O(1)$. The water flow corresponding to the averaged $\langle\theta\rangle=\phi_{1} \times \theta_{1}+\phi_{2} \times \theta_{2}\left(\theta_{1}\right.$ and $\theta_{2}$ are the volumetric water content of macro- and microporosity domain, respectively) here can be considered as an unsaturated flow, since the averaged water content is smaller than the total porosity of the medium. In the numerical test cases, the unsaturated steady-state flow condition with $\theta_{1}>\theta_{2}$ could be seen when we have the hydraulic head condition $h=h_{1}\left(\theta_{1}\right)=h_{2}\left(\theta_{2}\right)$ on water retention curves of the two domains (Lewandowska et al. 2005; Jougnot et al. 2008). This unsaturated steady-state flux was imposed at the inlet, and a given pressure (atmospheric) is considered at the outlet. The transport of the solute is started when the steady-state flow condition is reached.

For the solute transport, different boundary conditions were applied for cases 1 and 2, whereas the initial condition is zero concentration for both cases. In case 1 , the pulse-like concentration of $1 \mathrm{~g} / \mathrm{L}$ within $1000 \mathrm{~s}$ was introduced in the medium. In case 2, the step-like solicitation was used with a solute concentration of $1 \mathrm{~g} / \mathrm{L}$ at the medium inlet. The concentration gradient is zero at the outlet boundary for both examples.

Direct numerical simulation of the fine scale model was carried out in a manner of solving the coupled flow and transport problems by using the finite element code COMSOL Multiphysics ${ }^{\circledR}$. The medium was discretized into 8718 triangular elements for the $2 \mathrm{D}$ examples. These choices are the results of a prior mesh sensitivity analysis. Using a computer with a processor of i5-5200U CPU @ $2.20 \mathrm{GHz}$ and RAM of $4 \mathrm{~GB}$, the computation time needed to obtain the fine scale solution was about $75 \mathrm{~s}$ for the $2 \mathrm{D}$ examples.

The two numerical test cases were presented here in order to assess different transport phenomena reproduced by the developed model. In particular, the pulse-like transport producing more complex mechanisms than the step-like transport was studied to study the interesting concentration evolution inside the micro-porosity domain (inclusions). 


\subsection{Numerical Simulation of the Homogenized Model}

\subsubsection{Governing Equations}

To simulate the test cases presented above by the double-porosity model (i.e. homogenized model), we consider the 1D macroscopic solute transport for the macro-porous domain, which is coupled with the $2 \mathrm{D}$ transport problems in the micro-porous domain (circular inclusions). According to their local geometry, these 2D local problems can be transformed into 1D ones in cylindrical coordinates.

According to Eqs. (73)-(75), the homogenized model can be rewritten as follows:

$$
\begin{gathered}
\phi_{1} \frac{\partial\left(\theta_{1} C_{1}\right)}{\partial t}=\frac{\partial}{\partial z}\left(D_{\text {eff }} \frac{\partial C_{1}}{\partial z}-\langle v\rangle C_{1}\right)-\frac{1}{|\Omega|} \int_{\Gamma} D_{2} \frac{\partial C_{2}}{\partial r} \mathrm{~d} \Gamma \\
\frac{\partial\left(\theta_{2} C_{2}\right)}{\partial t}=D_{2}\left(\frac{\partial^{2} C^{2}}{\partial r^{2}}+\frac{1}{r} \frac{\partial C_{2}}{\partial r}\right) \\
C_{1}=C_{2} \quad \text { on the interface } \Gamma
\end{gathered}
$$

where $z$ and $r[\mathrm{~L}]$ are spatial coordinates, respectively. The initial and boundary conditions are applied for each case as follows:

- Case 1 corresponding to a solute transport by pulse-like injection:

$-t \leq 0$, for Eqs. (77)-(79):

$$
C_{1}=C_{2}=C_{0}=0
$$

- $t>0$, for Eq. (77), the third-type (Cauchy type) boundary condition was used to prescribe the concentration flux at the medium inlet $(z=0)$, whereas the second-type (Neumann type) for the medium outlet $(z=L)$ (van Genuchten and Parker 1984; Schoen et al. 1999; Cushman and Tartakovsky 2016):

$$
\begin{gathered}
z=0,0<t \leq t_{0}:\langle v\rangle C_{1}-D_{\text {eff }} \frac{\partial C_{1}}{\partial z}=\langle v\rangle C_{\text {inj }} \\
z=0, t>t_{0}:\langle v\rangle C_{1}-D_{\text {eff }} \frac{\partial C_{1}}{\partial z}=\langle v\rangle C_{0}
\end{gathered}
$$

where $C_{\mathrm{inj}}\left[\mathrm{M} / \mathrm{L}^{3}\right]$ is the concentration of the solute injected in the medium, $C_{\text {inj }}=1 \mathrm{~g} / \mathrm{L} ; C_{0}=0 ; t_{0}=1000 \mathrm{~s} ;\langle v\rangle=0.796 \times 10^{-7} \mathrm{~m} / \mathrm{s}$.

- $t>0$, for Eq. (78):

$$
\begin{gathered}
r=R: C_{1}(z)=C_{2}(r) \quad \text { on the interface } \Gamma \\
r=0: D_{2} \frac{\partial C_{2}}{\partial r}=0
\end{gathered}
$$

where $R[\mathrm{~L}]$ is the radius of the circular inclusions. 
- Case 2 corresponding to a solute transport by step-like injection:

The initial and boundary conditions of the test case 2 are all the same as the ones for the test case 1, except the boundary condition for Eq. (77) corresponding to a solute transport by step-like injection as follows:

$$
z=0, t>0:\langle v\rangle C_{1}-D_{\text {eff }} \frac{\partial C_{1}}{\partial z}=\langle v\rangle C_{\text {inj }}
$$

where $C_{\text {inj }}=1 \mathrm{~g} / \mathrm{L}$.

\subsubsection{Numerical Implementation}

Two main steps have to be followed to solve the macroscopic problem: (1) calculation of the effective parameter $D_{\text {eff }}$ by Eq. (76), after resolving the local boundary value problem given by Eqs. (58) and (59) and (43) for a given local geometry of the medium; (2) resolving the macroscopic boundary value problem of Eqs. (77)-(79) together with known initial and boundary conditions [Eqs. (80)-(85)]. A special algorithm is required for the coupling of the macroscopic concentration field with information on the concentration of the microporous domain. The effective parameter $D_{\text {eff }}$ is of $0.637 \times 10^{-9} \mathrm{~m}^{2} / \mathrm{s}$ for cases 1 and 2 . The value results from the resolution of the boundary value problem given by Eqs. (58) and (59) and (43) on a unit cell (period) of the double-porosity media (not presented in detail here, see in Tran Ngoc 2008).

The implementation strategy for the numerical resolution is the same as the one used for a similar two-equation model presented in Tran Ngoc et al. (2011). The macroscopic solution of the homogenized model will be obtained by solving a macroscopic 1D Eq. (77) coupled with a series of microscopic 1D local problems Eq. (78). We perform the computations at two coupled scales whose variables are as follows:

- the concentration $C_{1}(z)$ at the macroscopic domain is equal to the concentration $C_{2}$ at $r=R$ standing for all the micro-porous domain, Eq. (79);

- the diffusive flux [or source term in Eq. (77)]

$$
S=\frac{1}{|\Omega|} \int_{\Gamma} D_{2} \frac{\partial C_{2}}{\partial r} \mathrm{~d} \Gamma \quad(r=R)
$$

calculated on the interface of the micro-porous domain [from Eq. (78)] is inserted in the macroscopic equation [Eq. (77)].

This numerical implementation was performed in the environment of the COMSOL Multiphysics ${ }^{\circledR}$ software. The macroscopic domain is modelled geometrically 1D, whereas the 2D geometry (two independent variables $z$ and $r$ ) is used for the micro-porosity domain (domain 2). This is related to the strategy chosen here, which is to solve the 1D radial problem for all $z$ values, in order to facilitate the coupling with the macro-scale problem. The macroscopic domain 1 was uniformly discretized into 100 elements using $\Delta z=2 \times 10^{-4}$ $\mathrm{m}$. The discretization of the micro-domain $2(L \times R)$ representing the micro-porosity was performed by 1412 elements (612 triangular elements and 800 quadrilateral elements). The area close to the interface with the domain 1 is meshed finer. Due to $R \ll L$, we re-scaled 
the $r$ direction by factor 10 and thus got a mesh of 7050 elements (6250 triangular elements and 800 quadrilateral elements) in order to obtain more precise solutions. A mesh size sensitivity carried out by combining 50 or 100 elements in the domain 1 with 1412 or 7050 elements in the domain 2 showed a very slight difference in computation results. The simulations were only performed for a given value of the unsaturated steady-state water flow $\langle v\rangle(\theta)=0.796 \times 10^{-7} \mathrm{~m} / \mathrm{s}$ for cases 1 and 2 .

\subsection{Results and Comparisons}

\subsubsection{Case 1}

Figure 3 shows the concentration evolution curves (breakthrough curves) observed at the outlet of the medium for both the fine scale model (FSM) and the homogenized model (HM). A very good agreement between the two models was found with the coefficient of determination $R^{2}=0.9987$. It can be seen that the HM is capable to capture the early concentration breakthrough and the tailing effect of the non-Fickian transport. Note that $C(t)$ at $z=L$ of the fine scale model was computed by using a velocity-weighted average over the outlet surface (Golfier et al. 2011).

The concentration profiles at different times on an axis passing through the centre of the medium, obtained by the FSM and HM, are presented in Fig. 4. As expected, a strong variation of the concentration can be observed from the macro-porosity domain to the microporosity domain in the fine scale model solution. This shows the local non-equilibrium of the concentrations at short and intermediate times. The equilibrium concentration condition of the whole domain was reached after $t=6 \times 10^{5} \mathrm{~s}$. While the $C_{1}$ concentration profile of the HM reproduced very well the concentrations at each $z$ of all the macro-porosity domain, the $C_{2}$ concentration profile of the HM represented only for the concentrations at the centre of each inclusion ( $r=0$, micro-porosity domain), due to the numerical implementation manner employed. For comparing the concentrations at other positions in the micro-porosity domain, we can refer to Fig. 5. This figure corresponds to the computation of the concentration at each position $z$, by solving the radial problems given by Eq. (78). The concentration field is therefore plotted on the $(r, z)$ plane. This is used as a computation

Fig. 3 Comparison of the evolution of the concentration at the outlet of medium versus time obtained by the fine scale model (reference solution, dashed curve) and predicted by the homogenized model (bold curve) for the case 1

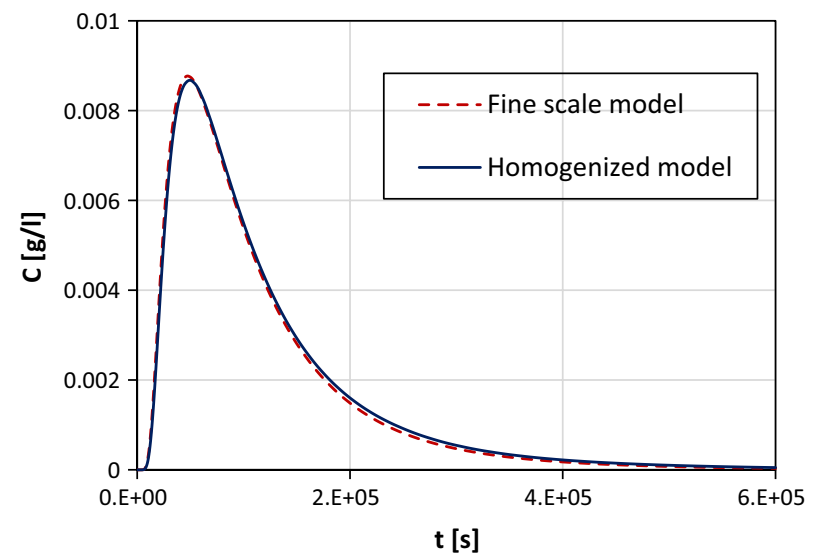


Fig. 4 Comparison between the concentration profiles along the axis in the centre of the medium at different times a $t=5 \times 10^{4} \mathrm{~s}$; b $t=8 \times 10^{4} \mathrm{~s}$; and $\mathbf{c} t=2 \times 10^{5} \mathrm{~s}$ obtained by the fine scale model (reference solution, dashed curve) and the homogenized model (bold curve for the concentration in the macro-porosity domain $C_{1}$ and thin curve for the concentration in micro-porosity domain $C_{2}$ ) for the case 1
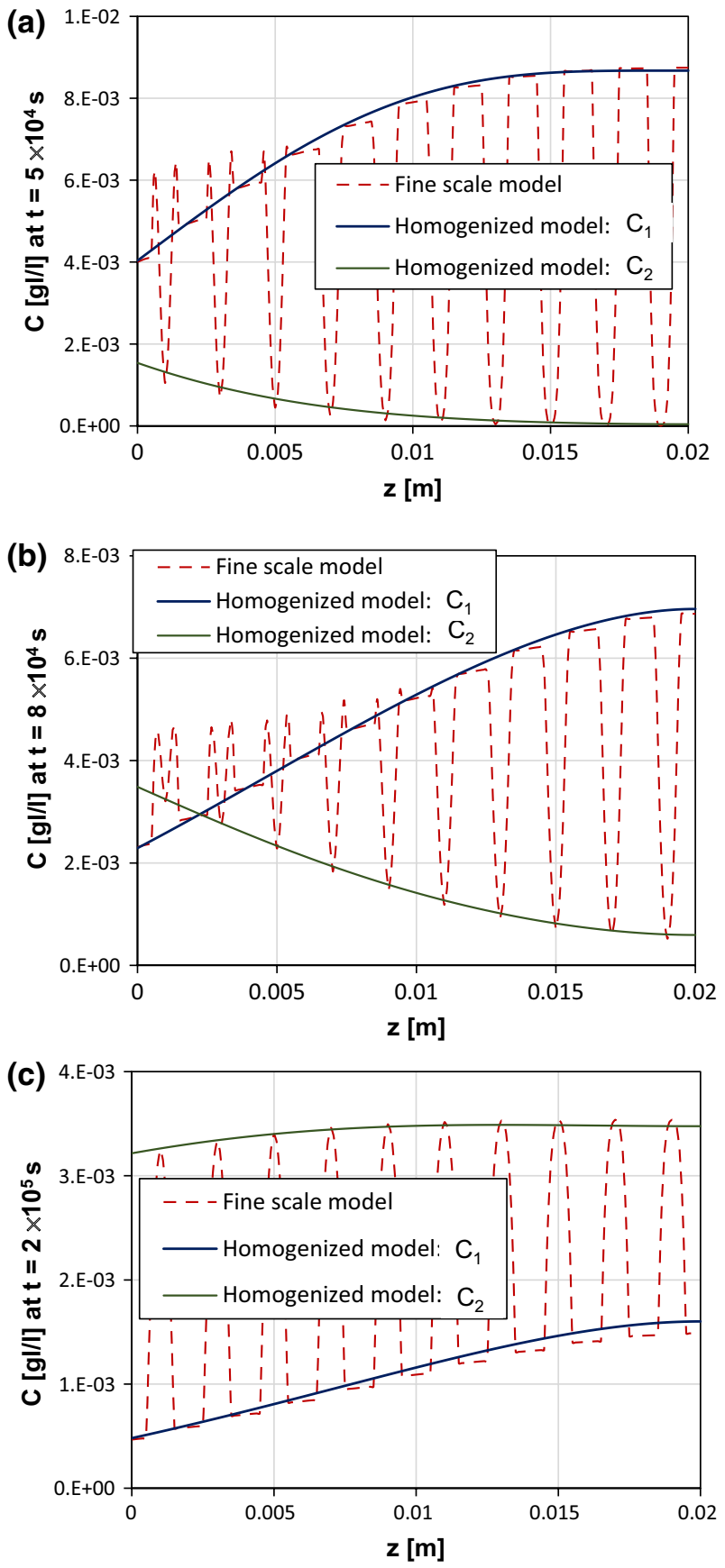
step for the coupling with the macroscopic model. It can be seen that the non-equilibrium solute transport processes occurring inside the micro-porosity domain from the inlet to the outlet at different times were very well captured by the HM. Note that the concentration profile $C_{2}$ presented in Fig. 4 is the concentrations on the cut line AB of Fig. 5. We can observe the high and low concentrations in the macro- and micro-porosity domains (Figs. 4a, b, 5a, b), respectively, for intermediate times $t$ corresponding to the vicinity of the concentration peak (Fig. 3). The inverse is observed for long times $t$ (Figs. 4c, 5c). It is interesting to see the coexistence of two mechanisms "solute flooding water and flooded by water" inside the micro-porosity domain along the medium expressed by the HM (Fig. 5b).

The numerical implementation allows to calculate the solute exchange term $S$ [Eq. (86)] between the macro- and micro-porosity domains. Figure 6 shows the evolution of the exchange term with time at selected $z$ in the medium. Due to the pulse-like solute input, we have $S>0$ when the solute enters into the micro-porosity domain from the macro-porosity domain and $S<0$ when the solute is released from the micro-porosity domain.

\subsubsection{Case 2}

The time evolution of the effluent concentration obtained by the FSM was very well reproduced by the HM Fig. 7. The $R^{2}=0.9985$ is as good as in case 1 . As case 1 , almost no solute exchange between the two porous domains is observed after $t=6 \times 10^{5} \mathrm{~s} \mathrm{(Fig.} \mathrm{8),} \mathrm{when}$ the concentration in all the medium reached at the injection concentration $\left(C_{\text {inj }}=1 \mathrm{~g} / \mathrm{L}\right)$ and the concentration tail tended to asymptotic (Fig. 7). By normalizing the same of concentration value as in case 1, the exchange source term $S$ with time is bigger than in case 1 by the fact of the continuous solute injection at the medium inlet. For this step-like injection, the only mechanism of the solute entering into the micro-porous domain from the macro-porous domain occurs, $S>0$ (Fig. 8).

Figure 9 presents the concentration profiles inside the inclusions of the double-porosity medium. Once again, in this case, it can be seen very clearly that the solute started to introduce in the inclusions at the earlier times (Fig. 9 a) and filled in them along the medium at the final times (Fig. 9b).

\section{Discussion and Conclusion}

Using the asymptotic homogenization theory, a homogenized model (HM) for solute transport in double-porosity media under unsaturated steady-state water flow conditions was developed. The model is limited to cases with strong diffusivity contrast between the micro- and macro-porous domains. These could be considered as a particular case with a very tortuous micro-porous domain causing diffusion at the local scale and thus leading to a macroscopic model giving a breakthrough curve with a tailing effect, i.e. non-Fickian transport (Royer and Boutin 2012). According to the defined orders of the Péclet number for different transport regimes at the local scale, a series of the macroscopic models for the double-porosity medium can be derived similarly as those presented in Auriault and Adler (1995) and Auriault and Lewandowska (1996). 
The developed model was numerically verified by confronting with the fine scale model (FSM) for the test cases with different boundary conditions. The homogenized model showed a good capacity to describe the local non-equilibrium concentration prevailing in transport processes and therefore the tailing effect. In the application of the HM to numerical test cases, the calculated Péclet number was $P e_{1 \ell}=\frac{\ell v_{1 c}}{D_{1 c}}=\frac{\ell}{D_{1 c}} \frac{\langle v\rangle}{\phi_{1}}=0.2$ for the cases 1 and 2 and the characteristic diffusion time of the two cases was $t_{\mathrm{c}}=L^{2} / D_{1 \mathrm{c}}=O\left(10^{6}\right) \mathrm{s}$. This is much greater than the convective time $O\left(10^{4}\right) \mathrm{s}$ and allows to complete the local diffusion process in the whole medium (Figs. 3, 5). According to the magnitude of the imposed velocity, the effective longitudinal diffusion coefficient was at the same order of magnitude as the diffusion coefficient of the macro-porous domain $D_{1}$. The HM can be the "richest" model (Royer and Boutin 2012), applicable in these example cases, i.e. a diffusion-convection regime for the example tests. Moreover, the validity domain of the numerical examples was verified with $\varepsilon=\frac{\ell}{L}=\frac{0.002}{0.02}=0.1$ :

$$
\begin{gathered}
10^{-3}<<Q=\frac{D_{2 c}}{D_{1 c}}=O\left(\varepsilon^{2}\right)<<0.1 \\
0.1<<P e_{1 \ell}=O\left(\varepsilon^{0}\right)<<10 .
\end{gathered}
$$

While Eq. (88) is satisfied, we have $Q=O\left(10^{-4}\right)$, therefore not satisfying Eq. (87) when $D_{1 c}$ and $D_{2 c}$ are estimated at the same order of magnitude as $D_{1}$ and $D_{2}$. Further simulations satisfying the entire domain of validity would require a greater macroscopic length $L$ than $0.02 \mathrm{~m}$ for the numerical examples, say $\geq 0.08 \mathrm{~m}$, equivalent to 40 periods. The tailing effect may even occur for the case without contrast between transport properties (Royer and Boutin 2012). It must be noted that even for the numerical examples with a very low $Q$ ratio presented here, the developed model (HM) revealed to capture fully the non-Fickian transport, although one of the conditions of Eq. (22) is not verified (Eq. 87).

The implementation method used in this work for the $\mathrm{HM}$ is capable to simulate simultaneously both solute concentrations at two scales, using the FEM code COMSOL Multiphysics $^{\circledR}$. To confirm the entire validity of the developed model for all orders of magnitude estimates imposed [Eq. (22)], other 2D and 3D tests should be performed by reemploying this implementation. The latter allows to apprehend what happens inside the inclusions. By the fact that each position of the macro-domain is considered interactive with the micro-domain, a sufficient number of microstructure periods is required and thus also for the macroscopic length of the medium. This is consistent with the requirement of the small separation parameter $\varepsilon$ [Eq. (1)]. The numerical tool elaborated in this study can be used for other two-equation models and modified for other microstructure geometries to study solute transport in physical models of double-porosity media presented in the literature. This tool can be applied to reproduce experimental results of Zinn et al. (2004) for tracer transport in a two-domain medium with intermediate and high contrast in hydraulic conductivity, and especially the experiments by Dalla Costa (2007) mimicking solute transport in a fractured medium, using a double-porosity model chosen with appropriate $D_{2} / D_{1}$ contrast. 
(a) $\mathrm{t}=5 \times 10^{4} \mathrm{~s} \quad$ Fine scale model

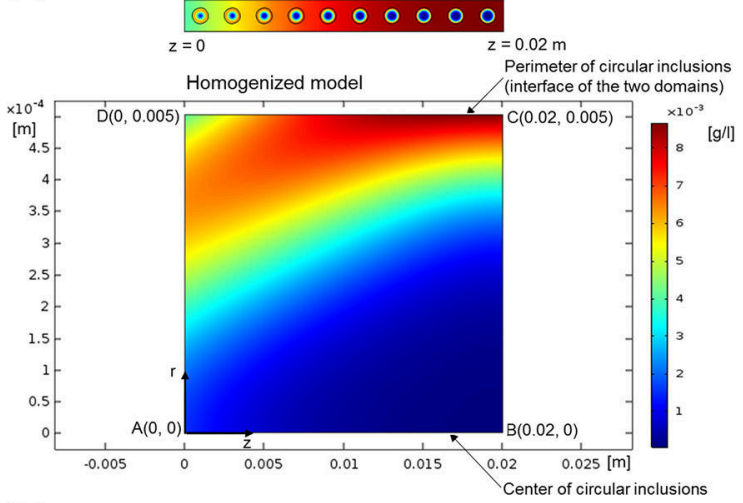

(b) $\mathrm{t}=8 \times 10^{4} \mathrm{~s} \quad$ Fine scale model

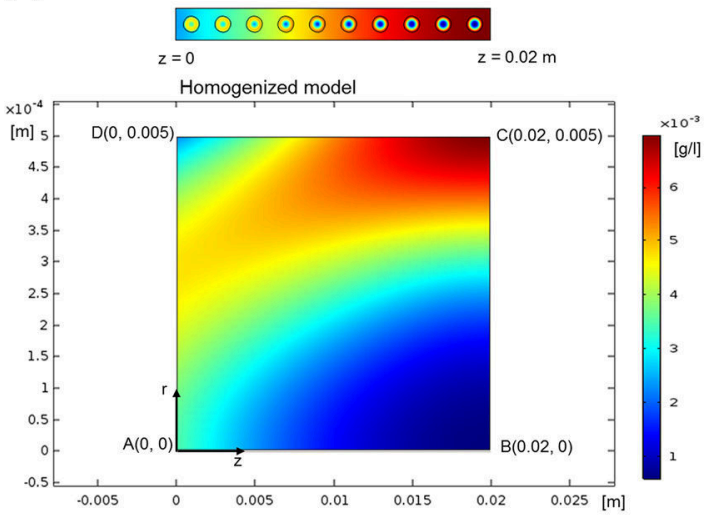

(c) $\mathrm{t}=2 \times 10^{5} \mathrm{~s} \quad$ Fine scale model

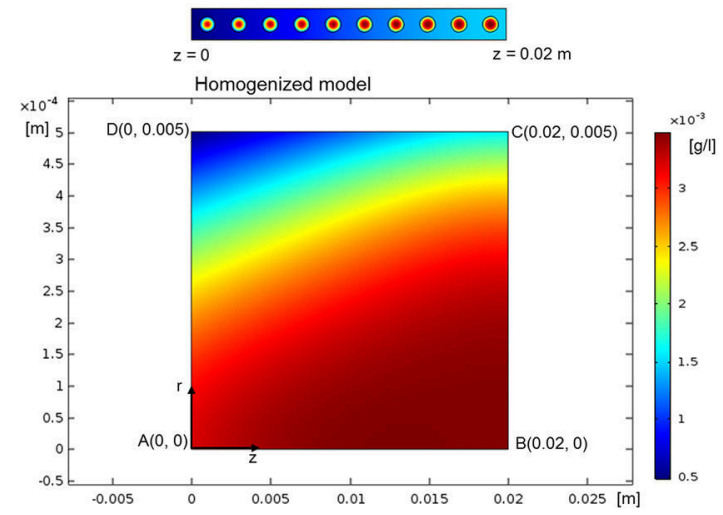

Fig. 5 Comparison between the concentration profiles of the whole medium (the fine scale model) and of the micro-porosity domain (inclusions) (the homogenized model) for the case 1. Each $z$ on AB line is implemented as the centre of the inclusions. CD line is the interface of the macro- and micro-porosity domains. $\mathrm{AD}$ or $\mathrm{BC}$ are the radius $R$ of the inclusions (to better visualize, the $r$ direction of the micro-porosity domain was enlarged by the factor $L / R$ ). To interpret, a concentration at a $z$ in the inclusion from the fine scale model (at the inclusion centre of the first period $z=0.001$ ), for example), to be compared with a concentration at the corresponding $r$ and $z$ from the homogenized model ( $r=0$ and $z=0.001$ : the concentration close to A) 
Fig. 6 Time evolution of the solute exchange term, normalized by a concentration of $2.21 \times$ $10^{-4} \mathrm{~g} / \mathrm{L}$ at $t=4 \times 10^{5} \mathrm{~s}$, between the macro- and micro-porous domains at different interface positions in the medium for the case 1

Fig. 7 Comparison of the evolution of the concentration at the outlet of medium versus time obtained by the fine scale model (reference solution, dashed curve) and predicted by the homogenized model (bold curve) for the case 2

Fig. 8 Time evolution of the solute exchange term, normalized by a concentration of $2.21 \times 10^{-4}$ $\mathrm{g} / \mathrm{L}$, between the macro- and micro-porous domains at different interface positions in the medium for the case 2
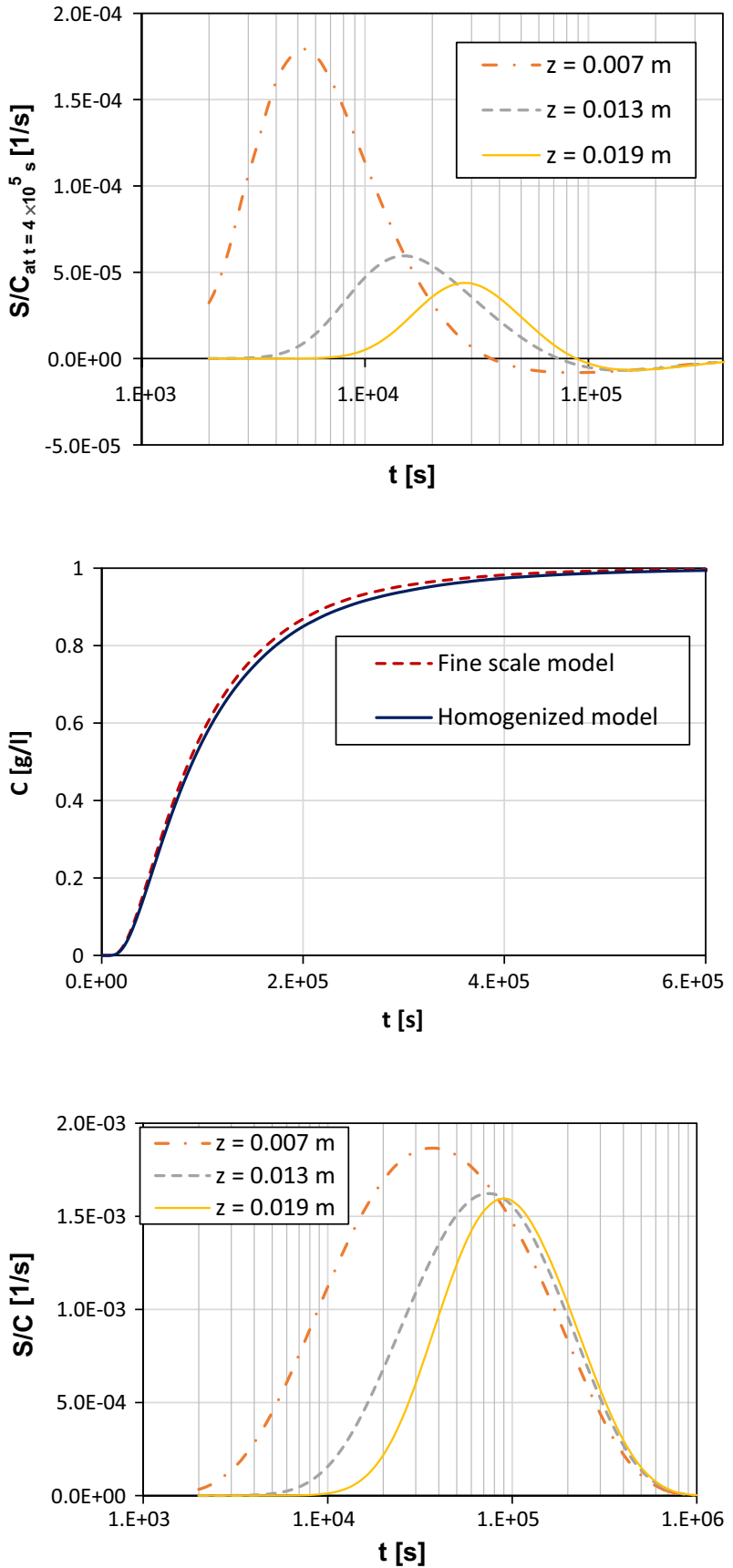


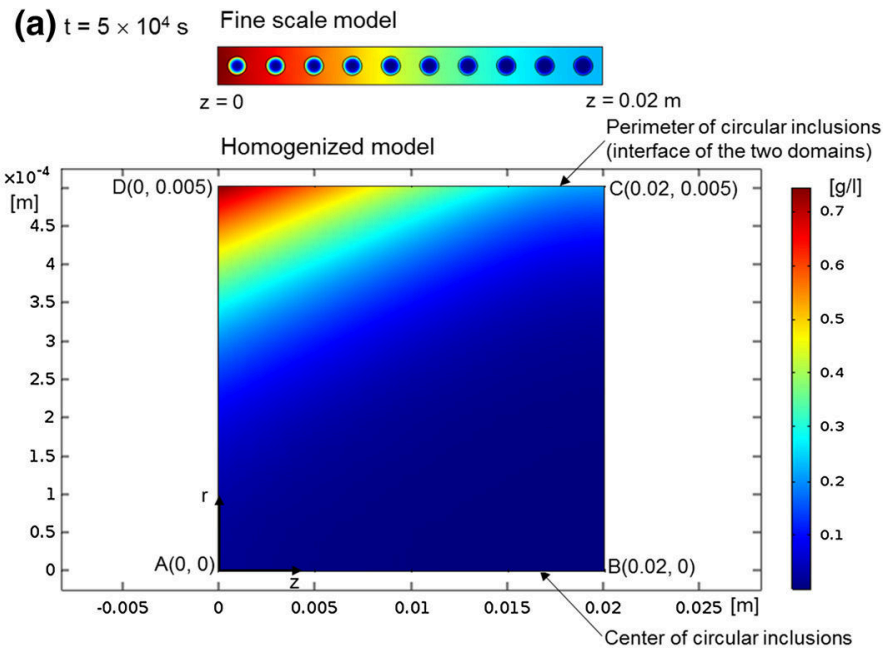

(b) $\mathrm{t}=2 \times 10^{5} \mathrm{~s} \quad$ Fine scale model

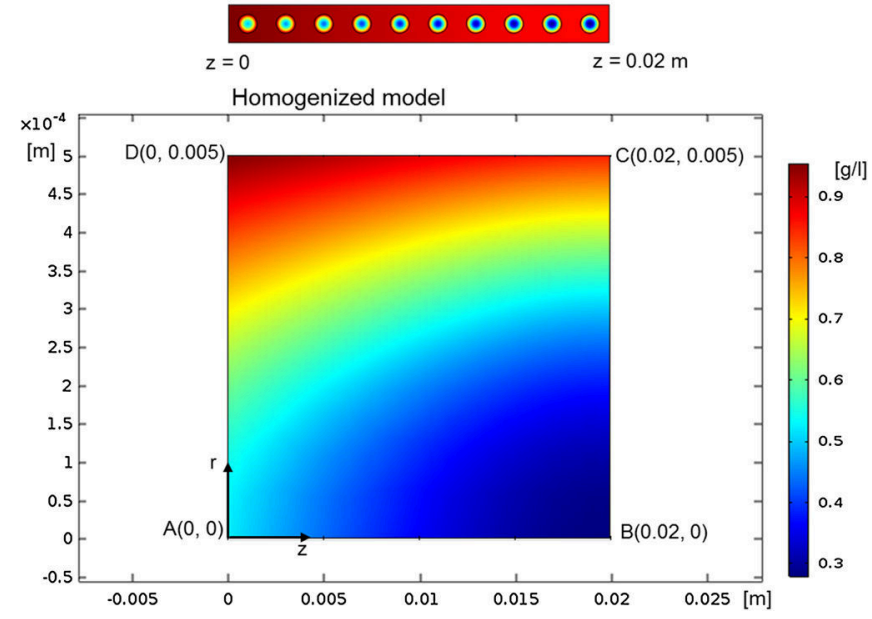

Fig. 9 Comparison between the concentration profiles of the whole medium (the fine scale model) and of the micro-porosity domain (inclusions) (the homogenized model) for the case 2. Each $z$ on $\mathrm{AB}$ line is implemented as the centre of the inclusions. CD line is the interface of the macro- and micro-porosity domains. $\mathrm{AD}$ or $\mathrm{BC}$ are the radius $R$ of the inclusions (to better visualize, the $r$ direction of the microporosity domain was enlarged by the factor $L / R$ )

Finally, the proposed homogenization and numerical simulation approach can be employed to investigate other physical couplings such as hydro-chemical with reaction or hydro-biological in heterogeneous porous media.

Acknowledgements This research is funded by Vietnam National Foundation for Science and Technology Development (NAFOSTED) under Grant Number 107.01-2015.25. The first author is greatly indebted to Prof. Jolanta Lewandowska, Université de Montpellier for supervising his Ph.D. thesis in which the method of homogenization has been comprehended. We would like to thank the anonymous reviewers for their valuable suggestions and comments. Funding was provided by NAFOSTED (Grant No. 107.01-2015.25). 


\section{References}

Ahmadi, A., Quintard, M., Whitaker, S.: Transport in chemically and mechanically heterogeneous porous media. V: two-equation model for solute transport with adsorption. Adv. Water Res. 22, 59-86 (1998)

Andricevic, R.: Effects of local dispersion and sampling volume on the evolution of concentration fluctuations in aquifers. Water Resour. Res. 34, 1115-1129 (1998)

Auriault, J.L.: Effective macroscopic description for heat conduction in periodic composites. Int. J. Heat Mass Transf. 26(6), 861-869 (1983)

Auriault, J.L.: Heterogeneous medium is an equivalent macroscopic description possible? Int. J. Eng. Sci. 29, 785-795 (1991)

Auriault, J., Adler, P.: Taylor dispersion in porous media: analysis by multiple scale expansions. Adv. Water Res. 18, 217-226 (1995)

Auriault, J.L., Lewandowska, J.: Non-Gaussian diffusion modeling in composite porous media by homogenization: tail effect. Transp. Porous Med. 21, 47-70 (1995)

Auriault, J.L., Lewandowska, J.: Diffusion/adsorption/advection macrotransport in soils. Eur. J. Mech. A/ Solids 15(4), 681-704 (1996)

Auriault, J.L., Boutin, C., Geindreau, C.: Homogenization of Coupled Phenomena in Heterogeneous Media. Wiley-ISTE, London (2009)

Barenblatt, G., Zheltov, I., Kochina, I.: Basic concepts in the theory of seepage of homogeneous liquids in the fissured rocks. J. Appl. Math. 24(5), 1286-1303 (1960)

Barenblatt, G.I.: On certain boundary-value-problems for the equations of seepage of liquid in fissured rocks. PMM 27(2), 348-350 (1963)

Bear, J.: Dynamics of fluids in porous media. Elsevier, New York (1972)

Bensoussan, A., Lions, J., Papanicolaou, G.: Asymptotic Analysis for Periodic Structures. North-Holland, Amsterdam (1978)

Berkowitz, B., Emmanuel, S., Scher, H.: Non-Fickian transport and multiplerate mass transfer in porous media. Water Resour. Res. 44, W03402 (2008)

Berkowitz, B., Scher, H.: Exploring the nature of non-Fickian transport in laboratory experiments. Adv. Water Res. 32, 750-755 (2009)

Berkowitz, B., Scher, H., Silliman, S.E.: Anomalous transport in laboratory-scale, heterogeneous porous media. Water Resour. Res. 36, 149-158 (2000)

Brassart, L., Stainier, L.: Effective transient behaviour of heterogeneous media in diffusion problems with a large contrast in the phase diffusivities. J. Mech. Phys. Solids 124, 366-391 (2019)

Cherblanc, F., Ahmadi, A., Quintard, M.: Two-medium description of dispersion in heterogeneous porous media: calculation of macroscopic properties. Water Resour. Res. 39(6), 1559-1579 (2003)

Cherblanc, F., Ahmadi, A., Quintard, M.: Two-domain description of solute transport in heterogeneous porous media: comparison between theoretical predictions and numerical experiments. Adv. Water Res. 30, 1127-1143 (2007)

Coats, K., Smith, B.: Dead-end pore volume and dispersion in porous media. Soc. Pet. Eng. J. 3, 73-84 (1964)

Cremer, C.J.M., Neuweiler, I., Bechtold, M., Vanderborght, J.: Solute transport in heterogeneous soil with time-dependent boundary conditions. Vadose Zone J. 15(6), 1-17 (2016)

Cushman, J.H., Tartakovsky, D.M.: The Handbook of Groundwater Engineering, 3rd edn. CRC Press, Boca Raton (2016)

Dagan, G.: Solute transport in heterogeneous porous formations. J. Fluid Mech. 145, 151-177 (1984)

Dagan, G., Russo, D., Bresler, E.: Effect of spatial variability upon subsurface transport of solutes from nonpoint sources. In: DeCoursey, D.G. (Ed.), International Symposium on Water Quality Modeling of Agricultural Nonpoint Sources Washington DC 1990, pp. 523-548. USDA-Agricultural Research Service

Dalla Costa, C.: Transferts d'un traceur en milieu poreux consolide et en milieu poreux fissure: Expérimentations et modélisations. Ph.D. thesis, Université Joseph Fourier-Grenoble, France (2007)

Dartois, A., Beaudoin, A., Serge, H.: Impact of local diffusion on macroscopic dispersion in threedimensional porous media. Comptes R. Mec. 346, 89-97 (2018)

Davit, Y., Quintard, M.: Technical notes on volume averaging in porous media I: how to choose a spatial averaging operator for periodic and quasi periodic structures. Transp. Porous Med. 119(3), 555-584 (2017) 
de Marsily, G., Delay, F., Gonçalvès, J., Renard, P., Teles, V., Violette, S.: Dealing with spatial heterogeneity. Hydrogeol. J. 13, 161-183 (2005)

de Vries, E.T., Raoof, A., van Genuchten, M.T.: Multiscale modelling of dual-porosity porous media; a computational pore-scale study for flow and solute transport. Adv. Water Res. 105, 82-95 (2017)

Doughty, C., Moridis, G.J.: Editorial to the special issue on the 2015 TOUGH Symposium. Transp. Porous Med 123(3), 455-456 (2018)

Fiori, A.: On the influence of local dispersion in solute transport through formations with evolving scales of heterogeneity. Water Resour. Res. 37, 235-242 (2001)

Fried, J.J., Combarnous, M.A.: Dispersion in porous media. In: Chen, V.T. (ed.) Advances in Hydroscience, vol. 7, pp. 169-282. Elsevier, New York (1971)

Gaudet, J.P., Jégat, H., Vachaud, G., Wierenga, P.J.: Solute transfer, with exchange between mobile and stagnant water through unsaturated sand. Soil Sci. Soc. Am. J. 41, 665-671 (1977)

Gaudet, J.-P., Jegat, H., Vachaud, G.: Étude du mécanisme des transferts d'eau et de soluté en zone non saturée avec prise en compte d'une fraction liquide immobile. Houille Blanche 3(4), 243-251 (1976)

Gensterblum, Y., Ghanizadeh, A., Cuss, R.J., Amann-Hildenbrand, A., Krooss, B.M., Clarkson, C.R., Harrington, J.F., Zoback, M.D.: Gas transport and storage capacity in shale gas reservoirs-a review. Part A: transport processes. J. Unconv. Oil Gas Resour. 12, 87-122 (2015)

Gerke, H.H.: Preferential flow description for structured soils. J. Plant Nutr. Soil Sci. 169, 382-400 (2006)

Gerke, H.H., van Genuchten, M.T.: A dual-porosity model for simulating the preferential movement of water and solutes in structured porous media. Water Resour. Res. 29, 305-319 (1993)

Golfier, F., Quintard, M., Cherblanc, F., Zinn, B.A., Wood, B.: Comparison of theory and experiment for solute transport in highly heterogeneous porous medium. Adv. Water Res. 30, 2235-2261 (2007)

Golfier, F., Quintard, M., Wood, B.: Comparison of theory and experiment for solute transport in weakly heterogeneous bimodal porous media. Adv. Water Res. 34, 899-914 (2011)

Ha, N.H., Bui, H.H., Nguyen, T.D., Le, N.S., Phan, N.T.: Development of a dual-porosity model for "Bach Ho" fractured basement reservoir. SOCAR Proc. 4, 12-20 (2015)

Hornung, U.: Homogenization of miscible displacement in unsaturated aggregated soils. In: Dynamical Systems. St. Petersburg (1991)

Huyakorn, P., Lester, B.H., Mercer, J.W.: An efficient finite element technique for modeling transport in fractured porous media. 1. Single species transport. Water Resour. Res. 19(3), 841-854 (1983)

Jankovic, I., Fiori, A., Dagan, G.: The impact of local diffusion on longitudinal macrodispersivity and its major effect upon anomalous transport in highly heterogeneous aquifers. Adv. Water Res. 32, 659-669 (2009)

Jarvis, N., Koestel, J., Larsbo, M.: Understanding preferential flow in the vadose zone: recent advances and future prospects. Vadose Zone J. 15(12), 1-11 (2016)

Jougnot, D., Lewandowska, J., Gotteland, P., Revil, A.: Hydraulic conductivity of unsaturated double porosity geomaterials. In: The 11th Baltic Sea Geotechnical Conference "Geotechnics in Maritime Engineering". Gdansk, Poland (2008)

Köhne, J.M., Köhne, S., Šimůnek, J.: A review of model applications for structured soils: a) water flow and tracer transport. J. Contam. Hydrol. 104, 4-35 (2009)

Lewandowska, J., Szymkiewicz, A., Burzynski, K., Vauclin, M.: Modeling of unsaturated water flow in double-porosity soils by the homogenization approach. Adv. Water Res. 27, 283-296 (2004)

Lewandowska, J., Szymkiewicz, A., Gorczewska, W., Vauclin, M.: Infiltration in a double-porosity medium: experiments and comparison with a theoretical model. Water Resour. Res. 41(2), 1-14 (2005)

Lewandowska, J., Tran Ngoc, T.D., Vauclin, M., Bertin, H.: Water drainage in double porosity soils: experiments and micro-macro modelling. ASCE J. Geotech. Geoenviron. Eng. 134(2), 231-243 (2008)

Majdalani, S., Chazarin, J.P., Delenne, C., Guinot, V.: Solute transport in periodical heterogeneous porous media: importance of observation scale and experimental sampling. J. Hydrol. 520, 52-60 (2015)

Mikelic, A., Rosier, C.: Modeling solute transport through unsaturated porous media using homogenization I. Comput. Appl. Math. 23(2-3), 195-211 (2004)

Ngien, S.K., Rahman, N.A., Bob, M.M., Ahmad, K., Sa'ari, R., Lewis, R.W.: Observation of light nonaqueous phase liquid migration in aggregated soil using image analysis. Transp. Porous Med 92, 83-100 (2012)

Ngo, T.S.: Kỳ tích tìm dầu ở tầng đá móng. Tia Sáng (in Vietnamese) (2002)

Niessner, J., Helmig, R.: Multi-scale modeling of three-phase-three-component processes in heterogeneous porous media. Adv. Water Res. 30(11), 2309-2325 (2007) 
Peng, Z., Duwig, C., Delmas, P., Gaudet, J.P., Gastelum Strozzi, A., Charrier, P., Denis, H.: Visualization and characterization of heterogeneous water flow in double-porosity media by means of X-ray computed tomography. Transp. Porous Med. 110(3), 543-564 (2015)

Peszynska, M., Showalter, R.: Multiscale elliptic-parabolic systems for flow and transport. Electron. J. Differ. Equ. 147, 1-30 (2007)

Portois, C., Boeije, C.S., Bertin, H., Atteia, O.: Foam for environmental remediation: generation and blocking effect. Transp. Porous Med. 124(3), 787-801 (2018)

Pruess, K., Narasimhan, T.N.: A practical method for modeling fluid and heat flow in fractured porous media. In: Paper Presented at the Sixth SPE Symposium on Reservoir Simulation, New Orleans, Louisiana (1982)

Quintard, M., Whitaker, S.: Two-phase flow in heterogeneous porous media: the method of large scale averaging. Transp. Porous Med. 3, 357-413 (1988)

Richards, L.: Capillary conduction of liquids through porous medium. Physics 1, 318-333 (1931)

Royer, P., Auriault, J.L., Boutin, C.: Macroscopic modeling of double-porosity reservoirs. J. Pet. Sci. Eng. 16, 187-202 (1996)

Royer, P., Auriault, J.L., Lewandowska, J., Serres, C.: Continuum modelling of contaminant transport in fractured porous media. Transp. Porous Med. 49, 333-359 (2002)

Royer, P., Boutin, C.: Time analysis of the three characteristic behaviours of dual-porosity media. I: fluid flow and solute transport. Transp. Porous Med 95(3), 603-626 (2012)

Sanchez-Palencia, E.: Non-homogeneous media and vibration theory. Lecture Notes in Physics, vol. 127. Springer-Verlag, Berlin (1980)

Schoen, R., Gaudet, J.P., Elrickb, D.E.: Modelling of solute transport in a large undisturbed lysimeter, during steady-state water flux. J. Hydrol. 215, 82-93 (1999)

Šimůnek, J., Jarvis, N., van Genuchten, M.T., Gardenas, A.: Review and comparison of models for describing non-equilibrium and preferential flow and transport in the vadose zone. J. Hydrol. 272, 14-35 (2003)

Šimůnek, J., van Genuchten, M.T.: Modeling non-equilibrium flow and transport processes using Hydrus. Vadose Zone J. 7, 782-797 (2008)

Sternberg, S.P.K., Cushman, J., Greenkorn, R.A.: Laboratory observation of nonlocal dispersion. Transp. Porous Med. 23, 135-151 (1996)

Sudicky, E.: A natural gradient experiment on solute transport in a sand aquifer: spatial variability of hydraulic conductivity and its role in the dispersion process. Water Resour. Res. 22, 2069-2082 (1986)

Szymkiewicz, A.: Modelling Water Flow in Unsaturated Porous Media. Accounting for Nonlinear Permeability and Material Heterogeneity. GeoPlanet: Earth and Planetary Sciences. Springer-Verlag, Berlin (2013)

Szymkiewicz, A., Lewandowska, J., Angulo-Jaramillo, R., Butlanska, J.: Two scale modeling of unsaturated water flow in a double-porosity medium under axi-symmetric conditions. Can. Geotech. J. 45, 238-251 (2008)

Tejchman, M.: Solute transport through single and dual porosity media. Mathematical modelling by homogenisation and column experimental investigations. Ph.D. thesis, Université Joseph Fourier-Grenoble, France (2004)

Tran Ngoc, T.D.: Transport de solutés dans un milieu à double-porosité non saturé. Modélisation par homogénéisation et application. Ph.D. thesis, Université Joseph Fourier-Grenoble, France (2008)

Tran Ngoc, T.D., Lewandowska, J., Bertin, H.: Experimental evidence of the double-porosity effects in geomaterials. Acta Geophys. 62(3), 642-655 (2014)

Tran Ngoc, T.D., Lewandowska, J., Vauclin, M., Bertin, H.: Two-scale model of solute dispersion in doubleporosity unsaturated media: homogenization and experiments. Int. J. Numer. Anal. Meth. Geomech. 35, 1536-1559 (2011)

van Genuchten, M.T., Parker, J.C.: Boundary conditions for displacement experiments through short laboratory soil columns 1 . Soil Sci. Soc. Am. J. 48(4), 703-708 (1984)

van Genuchten, M.T., Wierenga, P.J.: Mass transfer studies in sorbing porous media: I. Analytical solutions. Soil Sci. Soc. Am. J. 40, 473-480 (1976)

Warren, J.E., Root, P.J.: The behavior of naturally fractured reservoirs. J. Soc. Pet. Eng. 3, 245-255 (1963)

Whitaker, S.: Diffusion and reaction in a micropore-macropore model of a porous medium. Lat. Am. J. Appl. Chem. Eng. 13, 143-183 (1983)

Whitaker, S.: The Method of Averaging. Springer, Dordrecht (1999)

Wu, T., Li, X., Zhao, J., Zhang, D.: Multiscale pore structure and its effect on gas transport in organic-rich shale. Water Resour. Res. 53, 1-13 (2017) 
Zinn, B., Meigs, L.C., Harvay, C.F., Haggerty, R., Peplinski, W.J., von Schwerin, C.F.: Experiments visualization of solute transport and mass transfer processes in two dimensional conductivity fields with connected regions of high conductivity. Environ. Sci. Technol. 38, 3916-3926 (2004) 